\title{
LA MOSAÏQUE À L'OCÉAN TROUVÉE À MAUBOURGUET (Hautes-Pyrénées)
}

\author{
par Catherine BALMELLE ef Sylvain DOUSSAU
}

Depuis une quinzaine d'années, les decourertes de vestiges d'habitats antiques n'ont cessé de se multiplier dans les Hautes-Pyrenées, territoire de lancienne Aquitaine, jadis considéré comme peu romanise, mais en fait jusqualor's incompletement exploré par les archéologues ${ }^{1}$. Ainsi, le long de la vallée de l'Adour, à Maubourguet, trois sites d'établissements ruraux comportant des mosä́ques ont-ils été dernierement reconnus : l'un à l'emplacement de l'église, l'autre rue du cimetirrę2, le troisième enfin au domaine de Saint-Girons où a été découvert en 1979 un pavement représentant le dieu Ocían, objet de notre étude ${ }^{3}$. Par ses caractéristiques techniques et ornementales, ce parement de Maubourguet, comme on le verra, doit étre rapproché dautres mosaïques de l'Aquitaine méridionale et tout naturellement de la mosaïque d'oréan mise au jour à la fin du xix e siècle a Montréal-du(iers, au lieu-dit Cilésia.

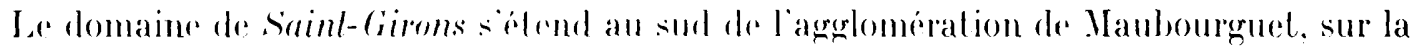
rive gauche de l'Alour, et cest à conviron $150 \mathrm{~m}$ du fleuvet que le plavement a été découvert fortuitement le 12 avril 1979, au cours de travaux agricoles. (iràce à une petite équipe composée de MII. R. Coquerel, s. Joussau ot J. Cinilhas, la mosaïque fut aussitòt entièrement régagée, en accord avee le propriétaire du terrain, I. Le Gentil. Laissée in silu,

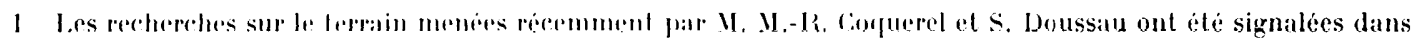
les chroniques de Gallia el dans les Bull. de la Sociele de Ramond Bagnerrs-de-Bigorre:, annees 1970 a 1973 . On consul-

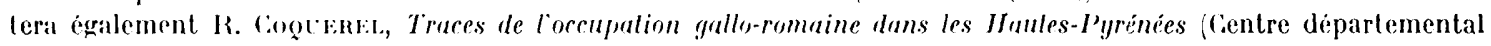
de la documentalion pedagogique, Tarbes, 197\%).

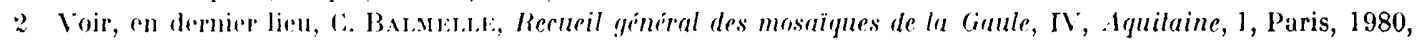

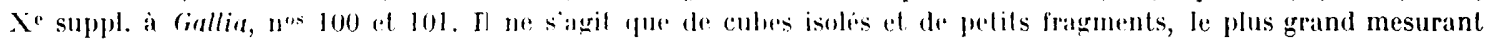
10 $\mathrm{cm} \operatorname{sur} 7 \mathrm{~cm}$.

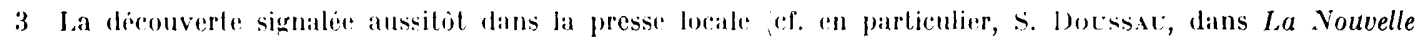
République des Pyrénes da :23 avril 1979, a cte menliomne dans les chroniques de Gallia, 38, 1980, p. 498, fig. 29.

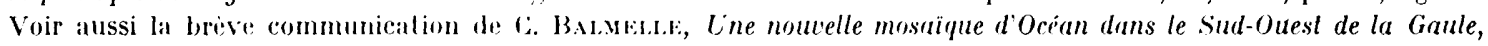
III Colloquio internazionale sul mosuico anlico, Racenna 6-10 sellembre 1980, liavenne, 19x:2.

4 Plan cadastral de 1973 , section C, parcelle $11 " 6: 2$. 

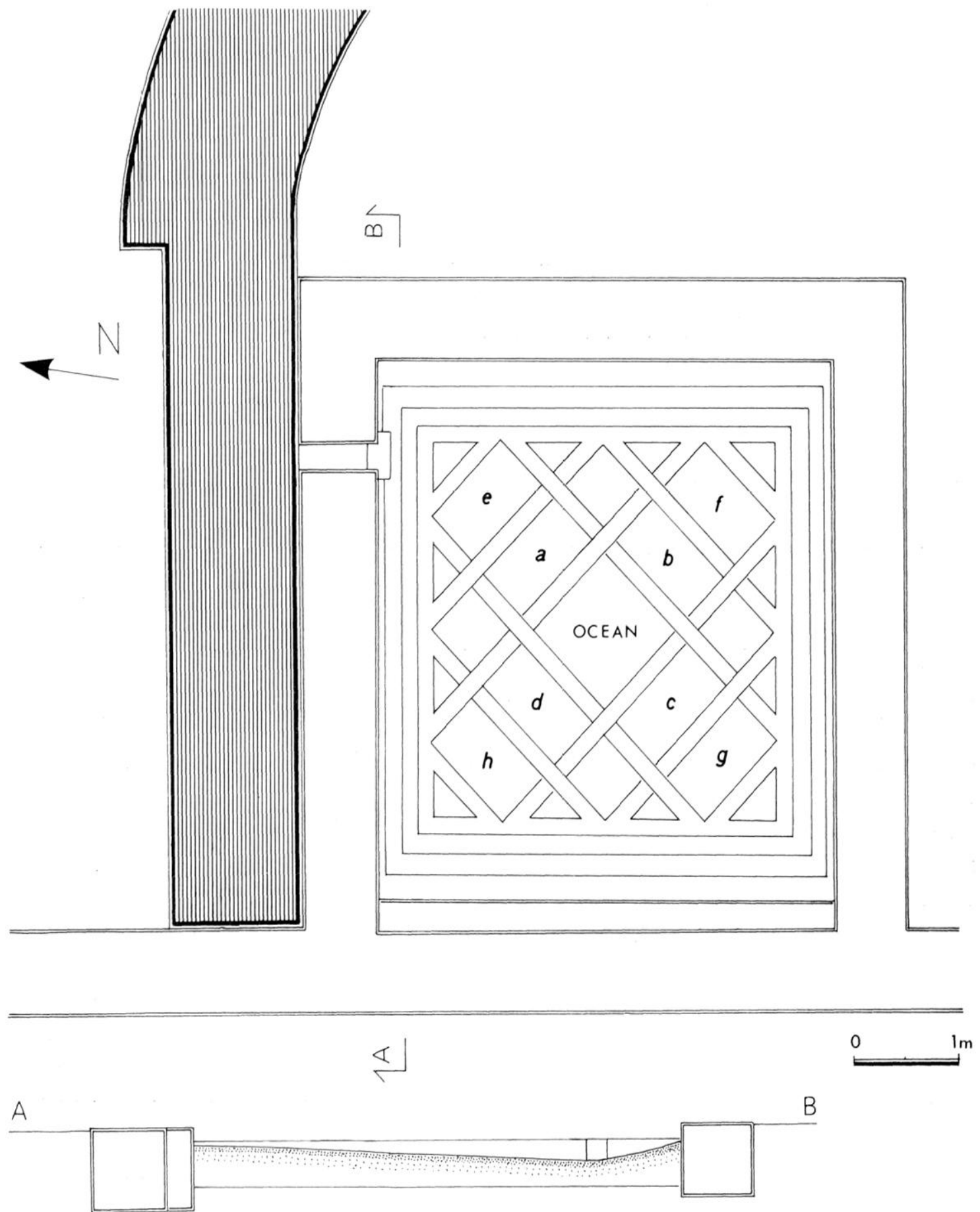

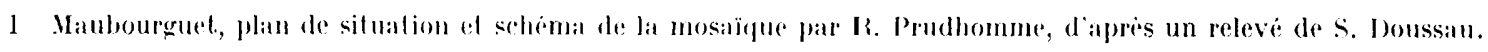

recouverte, elle a été inscrile en 1980 à löinventaire supplémentaire des Monuments historiques, à la suite des démarches menées par II. M. Labrousse, alors directeur des Antiquités historiques de Vidi-P'yrénées. 
La mosaïque courrait le sol d'un espare rectangulaire irrégulièrement implanté, d'environ $4.67 \mathrm{~m}$ sur $4,30 \mathrm{~m}$, délimité par des murs de $70 \mathrm{~cm}$ d'épaisseur, faits d'assises en galets liés au mortier. Le mur ouest ètait doublé à l'intérieur d'une banquette large de $25 \mathrm{~cm}$, constitué de galets et de nombreux fragments de tuiles, correspondant semble-t-il, à l'aménagement d'une marche. Aucune trace de seuil n'a été repérée; rependant, la présence de la banquette. l'orientation de la tête du dieu a l'ouest permettent de situer avec une certaine vraisemblance l'accès a la construction par le coté occidental (fig. 1). On notera qüintérieurement. les murs étaient enduits par endroits d'une couche de mortier rose, épaisse de $2 \mathrm{~cm}$; quelques fragments de plaque de marbre recueillis au cours du dégagement de la mosaïque laissent supposer que les parois verticales portaient, au moins a la base, un revetement de marbre.

Le pavement, couvert de concretions calcaires en divers points, etait relativement bien conservé (un dixième anviron de sa superficie détruit) (fig. 2 ): ce qui peut paraitre surprenant si l'on songe qu il se trouvait a $15 \mathrm{~cm}$ du sol actuel. En fait, il devait sa conservation à un pigeonnier qui le recourait jusque vers 1930. Attestée dés le Xvire siècle, la construction avait été implantée sur les murs entourant la mosaïque, assurant ainsi sa protection au milieu des terres cultivés. Comme le montre la coupe (fig. 1), le tapis présentait une dénivellation de $20 \mathrm{~cm}$ entre les points $A$ et $B$. La pente était nettement orientée vers une rigole d'evacuation qui passait dans le mur nord. Lne petite plaque de marbre violet, en forme de $T$, était scellée dans le pavement, encastrée dans le mur et déborlant légèrement au-dessus de l'évacuation (fig. 3). On signalera tontefois qu'aucune trace d'amenée d'eau n'a été repérée aux abords de la construction.

Il est évident que l'aménagement architectural de l'espace suggère d'y voir un bassin ; le décor de la mosaïque, à dominante de thèmes aquatiques, comme on le verra, était ainsi parfaitement arlapté a la fonction de la pièce.

Avant d'aborder l'étude létaillée du pavement, il importe de préciser qu'il n'y a pas eu de fouille systématique at que le matériel recueilli en surfare n'apporte aucune donnée rhronologique préciscte. Toutefois, en ce qui roncerne l'histoire du sile, plusieurs faits méritent d'être signalés. Premièrement, on notera que le mur ouest du bassin se prolongeait sur une dizaine de mètres vers le nord et sur 0,50 m vers le sud, et qu'au-delà, il était démoli jusqu'a la hase des fondations; aucune sulstruction napparaissait dans le prolongement des autres murs du bassin. I)ifférentes raisons pewrent expliquer ces destructions qui ont épargné le bassin protégé. a-t-on vu. par le pigeonnier : d'une part, la mise en culture du site dès le Voyen Age. d'autre part, l'usage de récupérer des débris de mortier de chaux pour l'amendement des terres. On indiquera également que le mur nord du bassin

5 Parfailement localise sur le plan cadastral de lx21, ce pigeonnier est deja mentionne dans les terriers du

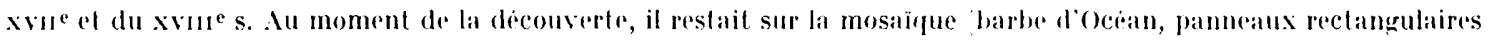
conligus; des traces de mortier d'un socle ell maconnerie qui devait apparlenir au pigeonnier.

6 Le materiel rencontri all-dessus de la mosaïgue consiste essentiellement en fragments de polerie du haut

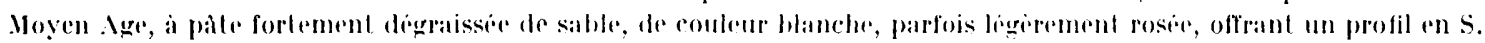

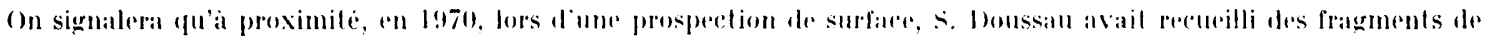
legulae et de ceramique commume gallo-romaine, ainsi que plusiemes fessons de ceramique du haul Ifogen Age. 

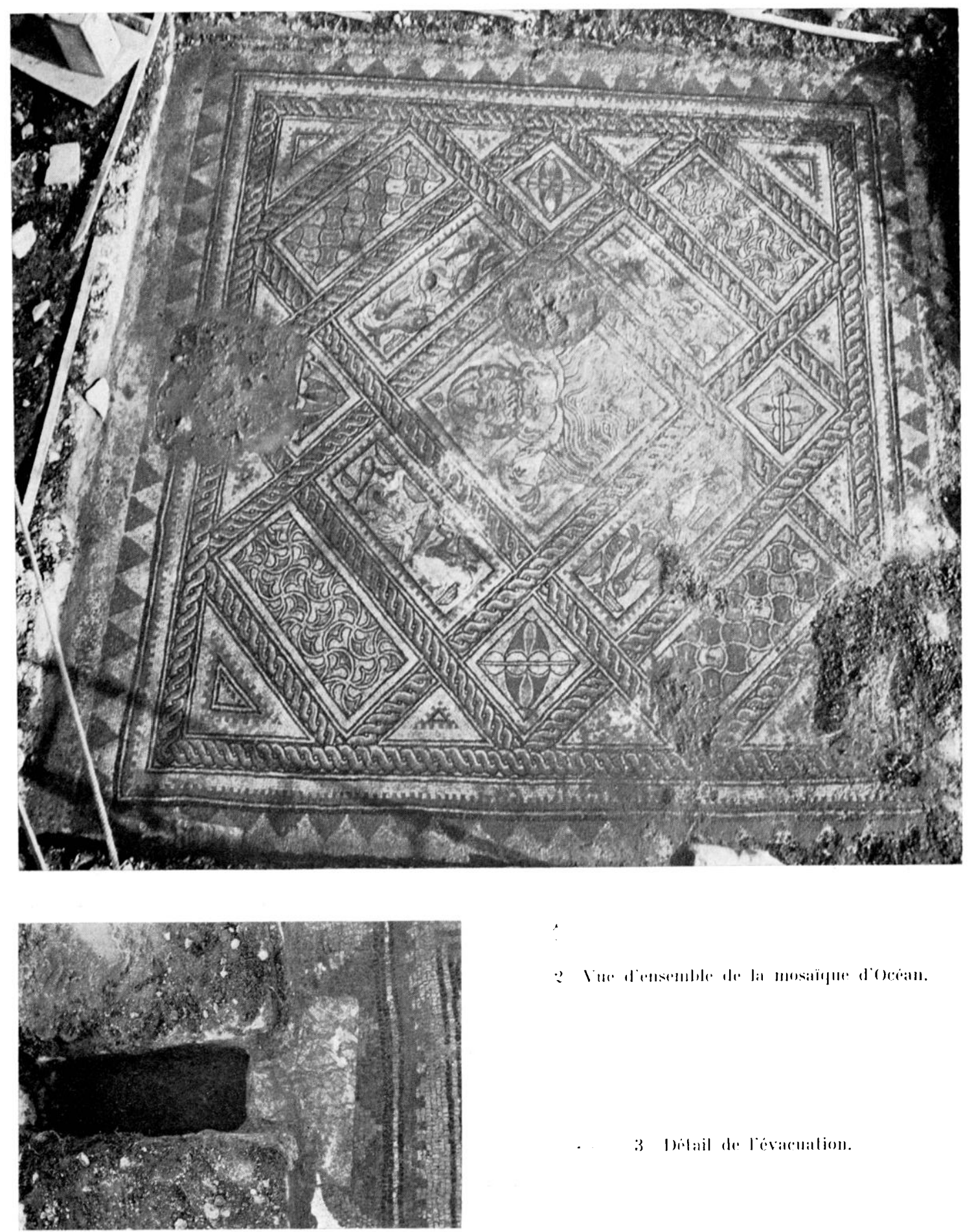

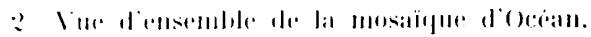

était doublé extericurement d'un mur postérivur. ipais de 1.83) m. so prolongeant à l'est

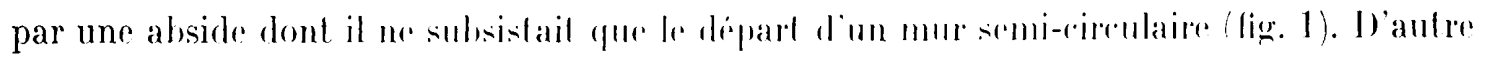

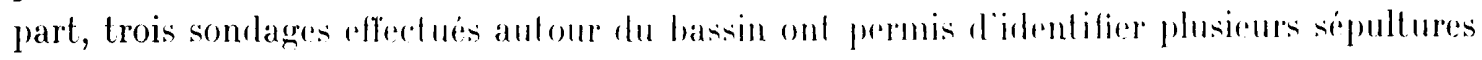
en pleine terre, dépourvurs dre mobilier, sans orientation précise ainsi que des fragments 


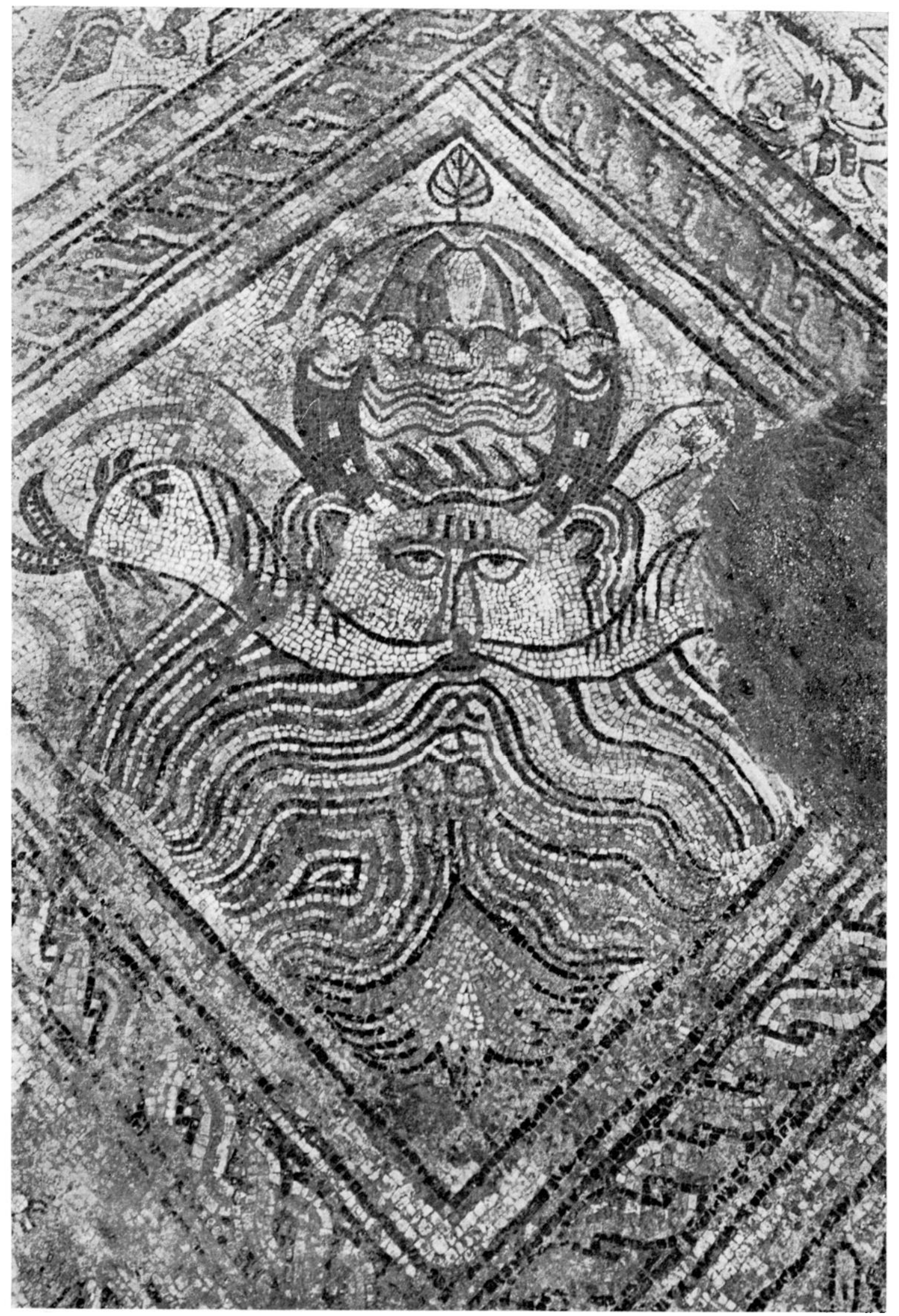

4 Tile docian. 
de sarcophages en marbre. En raison de l'abside orientée et des sépultures à proximité, on pourrait supposer qu'on se trouve en présence d'une église chrétienne?

Cies remarques sur le contexte architectural présentées, il convient d'examiner à présent la technique du pavement. Le mosaïste a fait usage de tesselles de formes irrégulières et de dimensions diverses (5 à $18 \mathrm{~mm}$ de côté), les éléments les plus fins étant réservés à l'exécution de la tête d'Océan (fig. 4). Comme dans de nombreuses mosaïques de cette partie de la (iaule, le mortier de jointoiement apparait nettement entre les cubes ${ }^{8}$. Quant aux matériaux employés, ce sont dans leur grande majorité des calcaires marneux ${ }^{9}$, mais on observe aussi de la terre cuite rouge et du marbre gris bleuté, blanc et mauve; cette dernière couleur, d'un emploi limité, se rencontre uniquement au centre du tapis, pour la tête du dieu.

Pour les couleurs de base, deux types principaux de calcaires sédimentaires ont été utilisés : d'une part, des calcaires à globigérines (blanc crème): d'autre part des calcaires à spicules (noir, gris, rose/rouge, jaune) dont les faciès varient sensiblement d'une couleur à l'autre. De tels calcaires n'ont pu être extraits des proches environs de Maubourguet; en revanche, ils pourraient provenir de la zone sous-pyrénéenne, au sud de Tarbes (région de Montgaillard). Toutefois, en ce qui concerne certaines catégories de calcaires à spicules, très durs (cf. noir et gris), on ne saurait exclure a priori l'hypothèse de galets de rivières.

Le marbre gris pourrail être un marbre du type de la haute vallée de la (iaronne, matériau assez souvent utilisé dans les mosaïques de l'Aquitaine méridionale ${ }^{10}$. Le marbre blanc et le marbre mauve (sans doute griotte) sont aussi vraisemblablement des marbres pyrénéens.

En ce qui concerne le chromatisme, on note une palette relativement réduite sur le pourtour du tapis : noir, gris, blanc crème parfois légèrement rosé, rouge (diverses nuances du rouge brique au bordeaux), rose clair et foncé, quelques rehauts de jaune. En revanche. dans le carré central et les carrés adjacents, les sujets figurés sont rendus au moyen d'une palette plus variée adjoignant du vert, du brun foncé, du mauve et du gris bleuté. En plusieurs secteurs, notamment dans le rectangle a (fig. 5) a), on remarque que les tesselles sont brûlées.

Les sujets figurés se caractérisent par un dessin schématique, linéaire et plat, des traits noirs cernant généralement les contours. Le tracé est sourent maladroit, les détails anat omiques sont simplifiés à l'extrème, entre autres dans les têtes des dauphins (fig. 4 et כj) ;

7 De plus, le toponyme du lieu-dit, saint-tirrons, atteste par les textes des 1233, n'exclut pas l'hypothese d'un édifice dédié a Cierontius, chrétien martyrisé en Novempopulanic à la fin de l'Antiquité ; sur ce martỵr, dont les origines et la vie sont en fait asse\% mal connues, voir par exemple Bibliolheca IIagiographica latina Antiquae et . Vediae Aetalis, ediderunt Socii Bollandiani, nos 3488-3489, cf. Acla Sanctorum, XrV, Maii, 2, p. 95 et 103-104.

8 Le supporl, examiné partiellement, présente un nucleus blanchàtre à grains fins et un rudus rose avec éclats de brique.

9 Les lames minces de plusienrs achantillons de lessedles effectures au laboratoire de petrographie de l'Universite de Paris VI, ont éte examinées par le Professeur J. Butanger. Qu'il soit ici vivement remercié. Notre reconnaissance va également au Professeur .I. 1)urand-1)elgal (laboraloire de géologie méditerranéenne, L'niversiti Paul-Sabatier, Toulouse', qui a ru la gentillesse d'orienter nos recherches sur les calcaires sedimentaires rencontres dans divers pavements du sud-()uest de la France.

10) Voir notamment Recueil, I1, 1, 1. 11. 

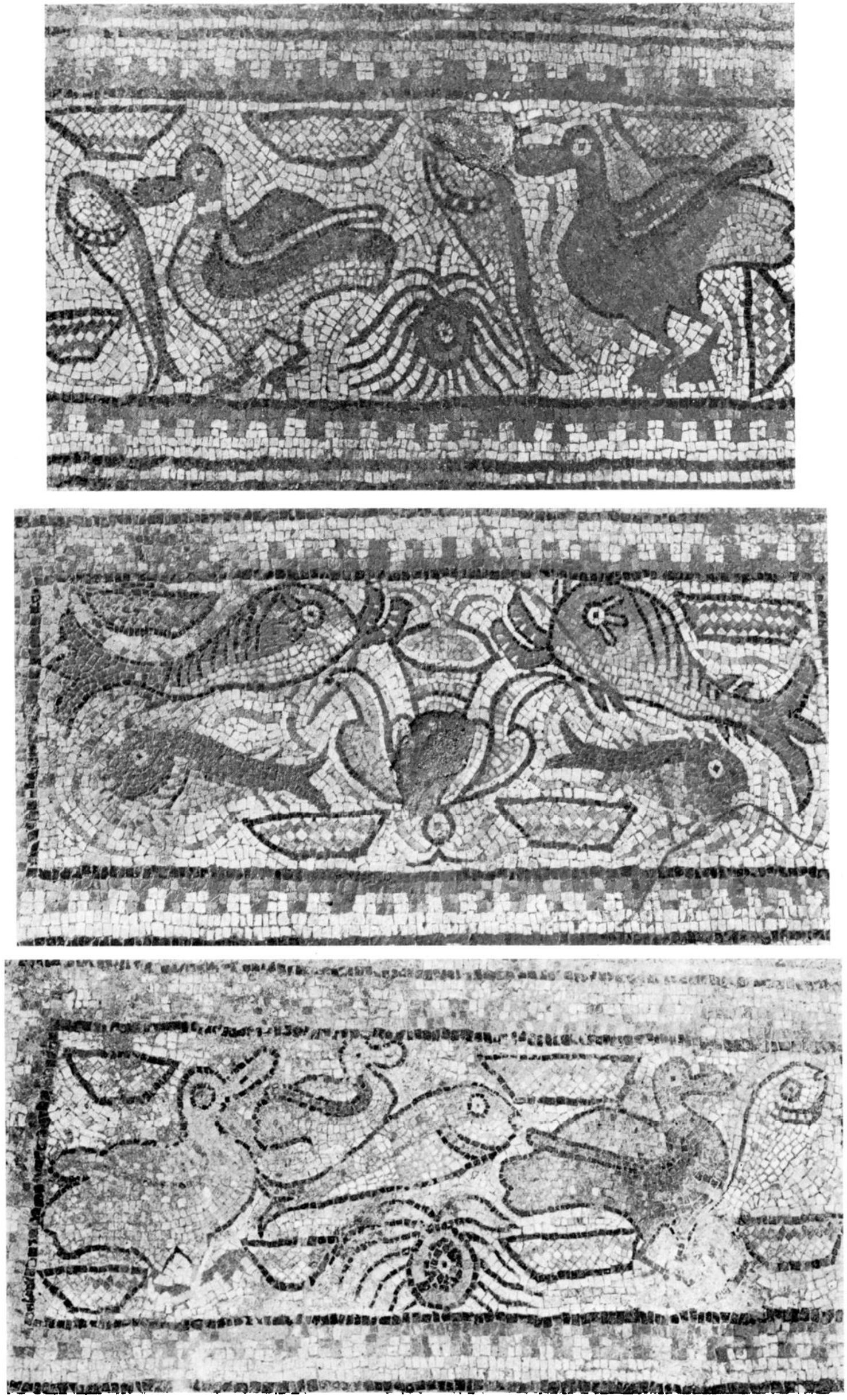

5) lo haul on bas : reclangles a, b of $c$. 
mais malgré tous ces défauts techniques, ces images demeurent étonnamment expressives et pleines de vie : il suffit d'observer en particulier le canard à l'allure guillerette qui décore l'un des rectangles (fig. 5) c). Pour ce qui est des motifs géométriques, on ne manquera pas de noter l'irrégularité des figures et les maladresses d'exécution, manifestes par exemple dans les peltes ( fig. 7 e). Ln dernier détail enfin mérite de retenir l’attention; il s'agit du tracé des bandes en lacis de tresses qui dessinent la trame. Bordées par un filet blanc entre deux filets noirs, les tresses, à l’intersection des bandes, sont nettement séparées l'une de l'autre, alors que le plus souvent les bandes tressées, simplement délimitées par un filet noir, forment un dessin continu"11. Le mème rendu en filet trichrome des bandes tressées en lacis, appliqué toutefois à une trame différente, se retrouve sur un pavement de la villa de Sorde-l'Abbaye (Landes) (fig. 6) ${ }^{12}$. Cette facon de dessiner les bandes en lacis ne constitue pas un procédé propre aux mosaïstes aquitains; des traits d'exécution analogues ont été repérés sur une série de pavements de l'Antiquité tardive, principalement aux ve et ${ }^{2}{ }^{e}$ siècles, dans l'ensemble dı monde méditerranéen ${ }^{13}$.

Le mosaïste avait à paver un espace architectural réduit. proche du carré (environ 4,67 m sur 4,30 m), aux dimensions irrégulières, comme on l'a v'u plus haut. Le systeme de composition géométrique choisi, de type centré et parfaitement symétrique, sadaptait à un cadre carré ct entraînait obligaloirement des raccords aux murs de largeur inégale. Le tapis décoré mis en placre. grosso modo carré $(1,20) \mathrm{m}$ de còté). fut ainsi séparé des murs par une bande de tesselles blanches (dlisposées en rangs perpendiculaires aux murs), de $5 \mathrm{~cm}$ au norl et au sud, de $21 \mathrm{~cm}$ à l'est et de $2.2,5 \mathrm{~cm}$ à $26 \mathrm{~cm}$ à l'ouest. Les différences de dimensions de l'espace architectural ont été aussi rattrapées en jouant sur la largeur de la borrlure (B0 a 46 (:m). Toutefois, eres irrégularités n'ont pu ètre reportées sur le plan (fig. 1).

11 (if. patr "ximple Recueil, II, 1, n० 161.

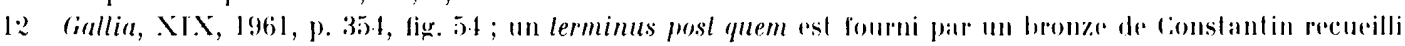
dans le nucleus du pavement. Precisons toutefois que lanalyse stylistique suggrererat une datation posterienre au milieu du rve s. I.e puban onde avec calices frifides, par exemple, dessine par deux sinusoïdes en filel triple, qui s'entrecroisent, se distingue nellement des rubans de ce lype rencont rés an a s. ; en revanche, les memes caracterisriques dans le trailement du ruhan se relrouvent sur des pavements du "Palais de Theodoric "a Ravenne (F. Berte,

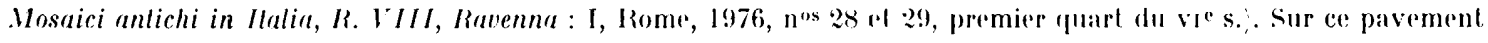
de la villa de Sorde-l'Ahbaye, voir ultipreurement, Recueil, IV, 2, en préparation.

13 On mentionnerai simplement quelques exemples parmi d'autres : Antioche (1). L.Ev, Anlioch Mosaic

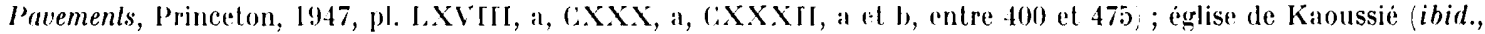
pl. CXIV, datee de 387 ; Cos, basiliques paleochreliennes is. PELEkswins, Corpus mostaicorum christianorum velusliorum pacimentorum : I, ciraecia Insularis, Thessaloniqur, 1974, pl. 2Х, 31, 34 à 37, 39, 42, ve/vie s.j; Loupian

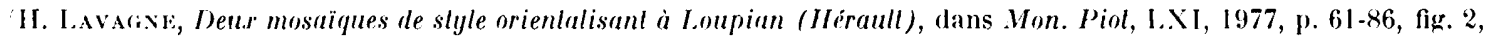
10, 16, 20, premiere moitie du ve s. ; Carthage, basiligue de Dermech II al salle voisine du baptistere souterrain

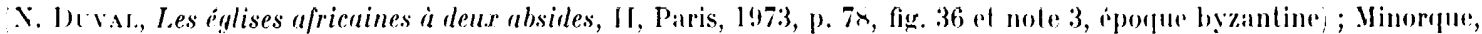

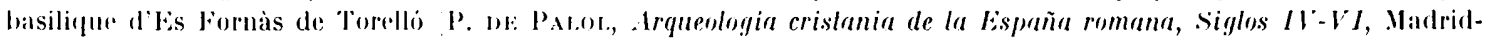

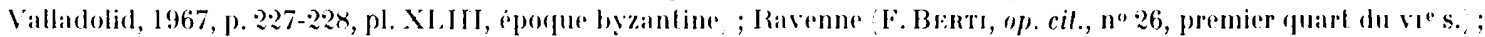
Naples, basilique de San 1.orenzo Maggiore R. Farmor, dans Calacombes el basiliques, Rome, 1978, p. 160, pl. X, a, 535-555). - Celte liste volontairement sálective nécessite queldques precisions : à l'exception d'un pavement d'antioche

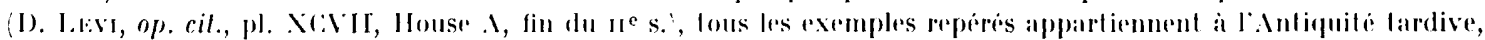

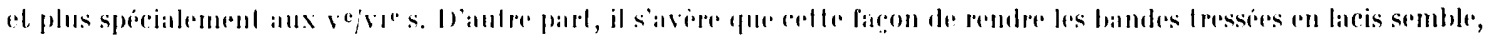

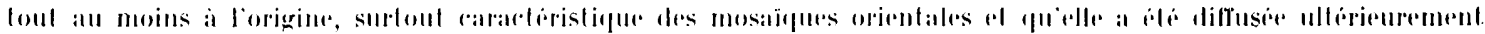

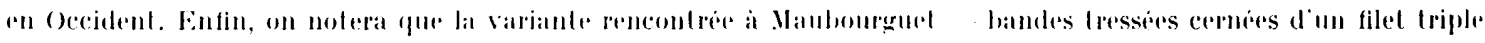

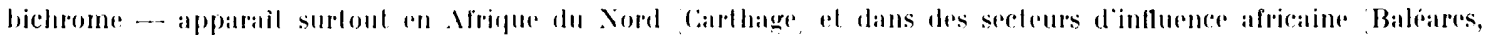
Italie du sud. 


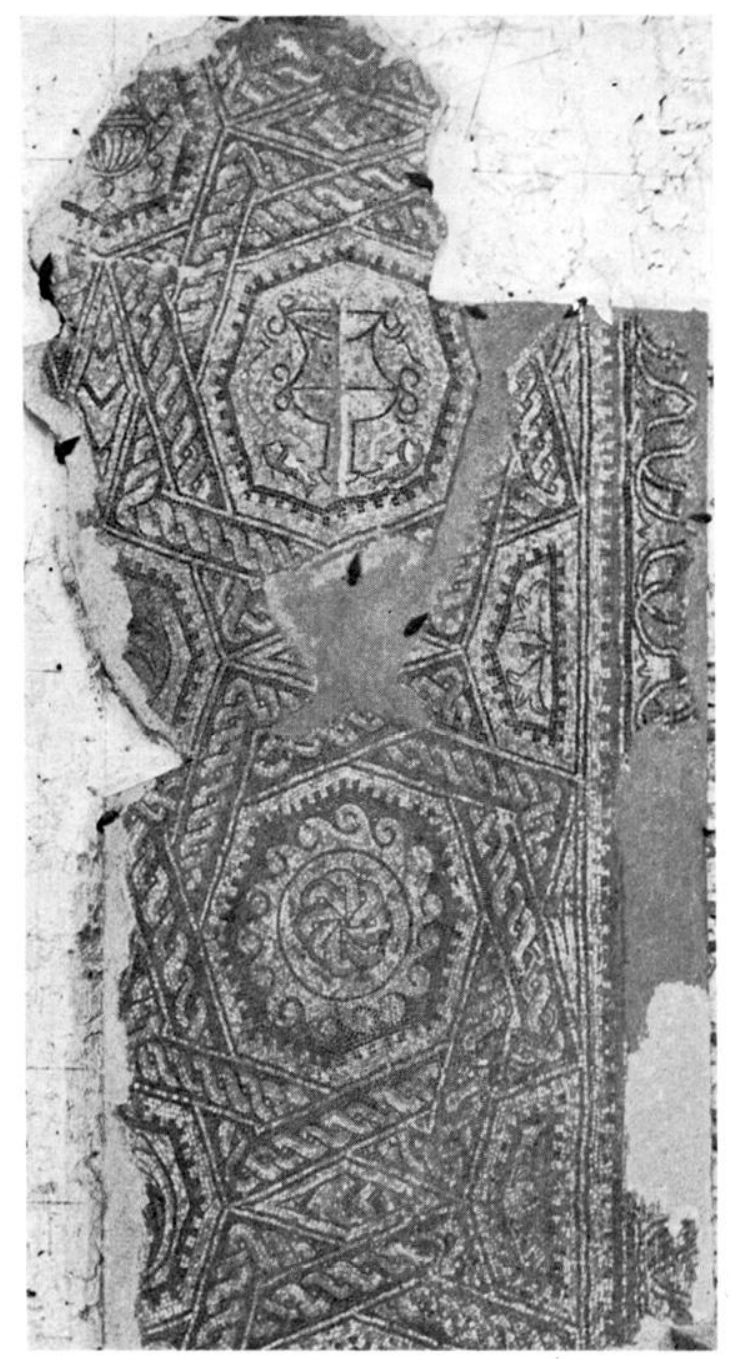

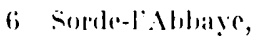
fragment du lapis avece eloiles de deux carres.

La bordure du tapis cist ainsi constitué (en allant de l'exterieur vers l'intérieur) : dents de scies dentelées réversibles, rouges et blanches ; filet double noir; filet blanc; filet noir ; filet double rouge à denticuless en quatre-tesselles, tournés vers l'inlérieur ; bande blanche; filet noir ; filet blane; filet noir ; tresse à deux brins (noir, rouge, jaune, blanc, noir et noir, gris, gris vert, blane, noir) sur fond blanc.

Le champ est organisé selon un système de composition centrée a plusieurs éléments, que l'on peut décrire de la manierre suivante : un grant carré sur la pointe inscrit, enlacé à une croix an sautoir, l'onsomble déterminant des paires de rectangles, des petits carrés sur' la pointe ainsi que des triangrles rectangles et isocèles sur le pourtour (fig. 1). Le schéma est traité en lacis de tresses a deux brins, de coloris irlentiques à ceux de la tresse entourant le champ (quelques varianles dans la répartition des couleurs).

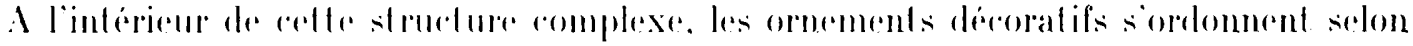

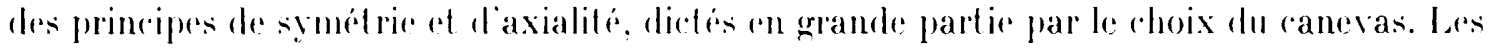
sujets aquatiques sont dominants : ()éan, dauphins, poissons, poulpes, canards, coquilles. 
S'y adjoignent des motifs géométriques aux lignes sinueuses, sur le pourtour. La lite du dieu Océan, au centre du champ, oceupant done une place privilégiée. est orientée vers l'ouest, tandis que les autres motifs figurés, répartis symétriquement dans les rectangles a, b. c, d (fig. 1). sont lisibles des angles du tapis ; le pavement s'offrait ainsi i la vue sous des angles multiples.

Carré central (96 cm de ròté): bordé à l'intérieur d'un filet rouge ol d'un lilet noir, il présente sur fond blanc, disposée selon la diagonale, une tète d'ocian, curieusement "coiffée» d'une coquille. aux rayons tournés vers le bas, sur laquelle se dresse une feuille cordiforme (lig. 4 ).

De part et d'autre du front du dieu. jaillissent deux grosses pinces de crustacé it dominante rouge (homard?) ainsi que deux antennes rouges : des lignes ondulées, érocquant sans doule les flols, sont disposées entre les pinces. Le visage démesurément large. se carar lérise par des oreilles bilobées et par un front bas de couleur mauve, orné de stries el, couronné par une sorte d'accolade au-dessus de larquelle sont figurés des trails obliques (chevelure?). () nolera igalement le ne\% long et informe d'où s'échappent symétriquement deux dauphins trìs stylisés (blancs et gris, cernes noirs, rehauts rouges), celui de droite partiellement ronservé. Je visige est encadré par une chevelure et une barbe abondantes, indifférenciées, rendues au moyen de lignes sinuenses (noir, blanc, rouge, roser, jaune, gris, brun. mauve) dont certaines dessinent des formes indistinctes. Wans la partie inférieure de la barbe est représenté. pointant vers l'angle du carré. un bouton de fleur trifide jaune el gris bleuté, orné de stries noires ${ }^{14}$.

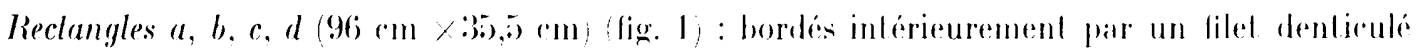
rouge et par un filet noir, ils montrent sur un fond clair parsemi de lignes ondulies, deux lypes de décor qui se répondent symétriquement. selon les diagonales : anards, poissons. poulpe (a. $\cdot$ )

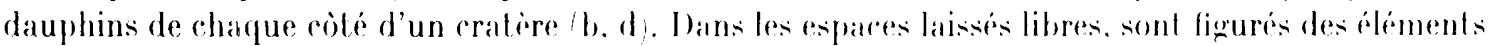
en forme de trapeze, rernés de noir. rendus au moven de lesselles posces sur la pointe faisant appairaitre des traits chenillés. Il est difficile de préciser la nature de ces elóments : les traits rhenilles rappellent l'un des procédés fréquemment utilisé par les mosinistes romains pour rendre l'eaun ${ }^{15}$. Toulefois. comple lenu de leur forme. de leur disposition el de leur emploi dans un contexte andailique, on pourrait songer à des plantes d'eau ou mieux encore a des paniers, ornements les uns et les autres souvent associés aux représentations de canards et de poissons ${ }^{16}$.

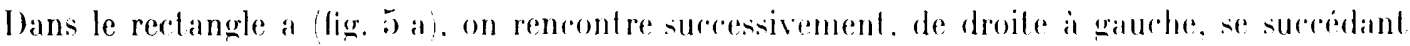
i la file : un canard au plumage rouge el jaune. l'ate soulignée en noir el gris. doti diun large bee rouge et de pieds palmés également rouges (sourhet? : un poisson banc el gris. se distinguant par

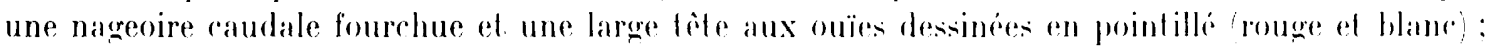

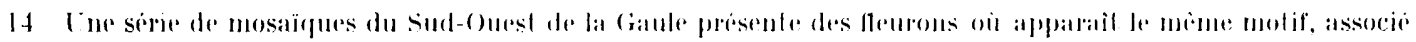

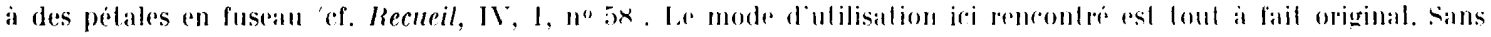

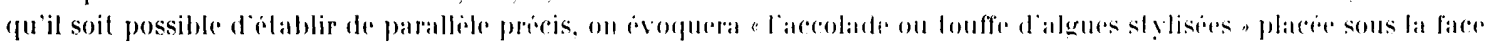

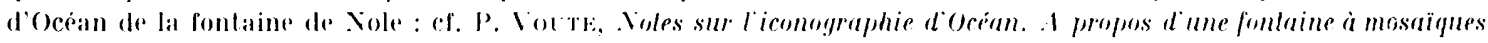

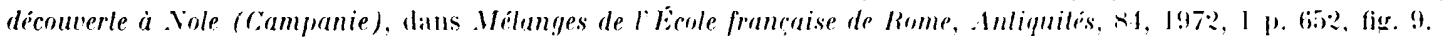

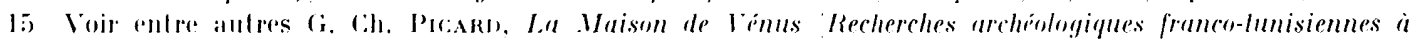
Mactar, T, Rome, 1977, p. 25 (.1 70, figr. :5).

16 Jat seconde hypothese nous parail aujourd hui plus vaisemblable, pell raison des lesselles sur la pointe qui pervent aussi suggeper une vannerie : cf. par exemple les paniers lemus far les amours pecherurs sur la mosäque

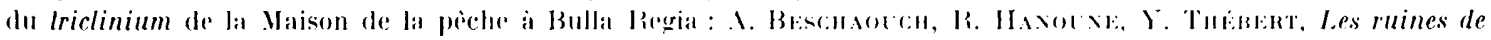

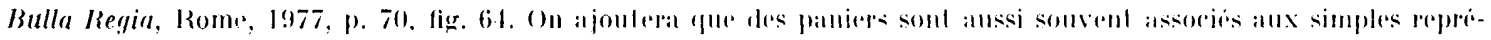

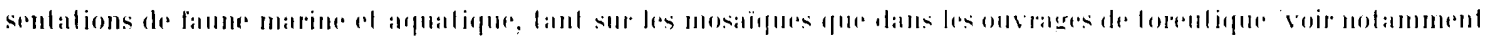

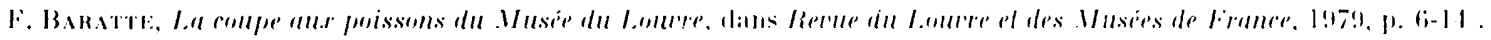

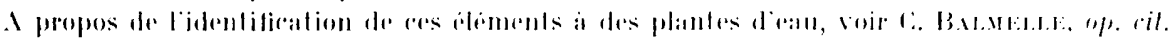



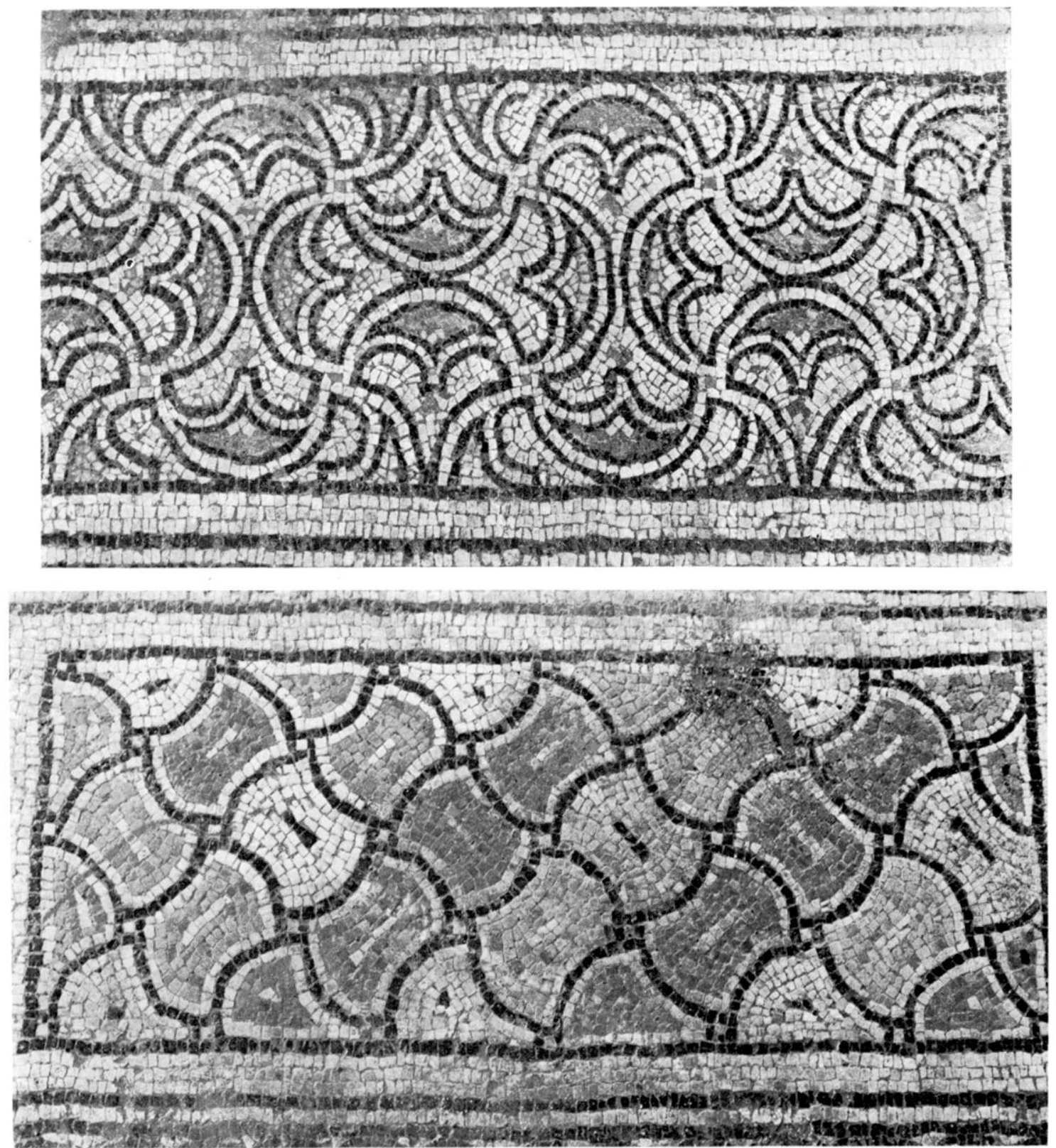

7 the hant en has : rectingle e, decori de peltes et rectangle f, decore d'osselets.

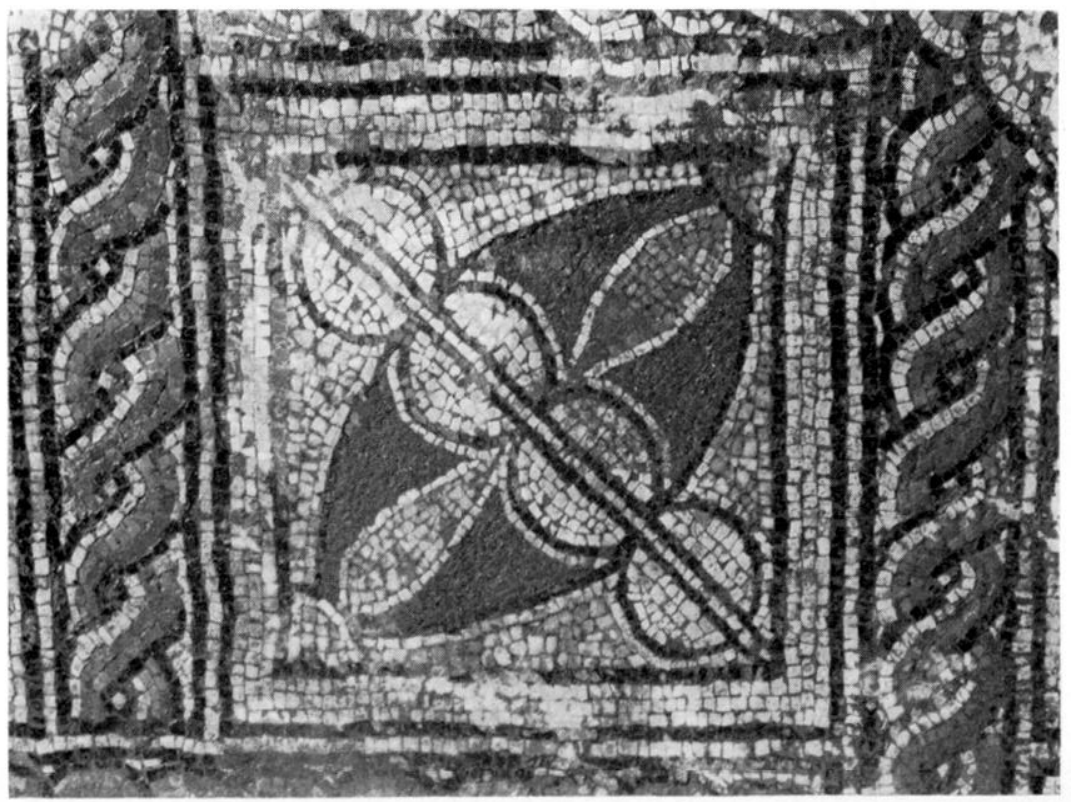

$x$ Deilail d'un peril carrie. 


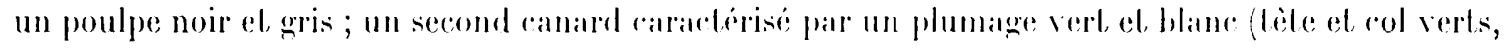

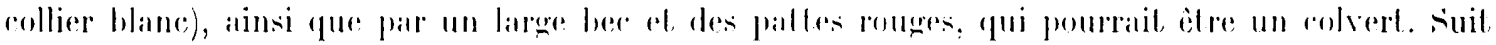
enfin un poisson analogue au premier, mais plus petit. L d's cantards sont representés de profil dans une attilude de marche; les poissons onl te corps dresié, la lète il la hauleur du bee des canards.

Dans le rectangle c (lig. jo c), on retrouve la mème alternance des éléments : canards à large bec et à pattes palmées (dont sans doute également un colvert), poissons et poulpes vers le milieu du panneau ; toutefois quelques variantes s'observent dans la disposition et le rendu des animaux. On notera ainsi deux canards còte a côte, l'un au plumage jaune saisi en marche, l'autre à dominante grise, plus petit, semblant nager, les patles dissimulées par un poisson gris bleuté à taches rouges, peut-être une truite. Le second poisson se remarque par sal couleur d'un blanc brillant.

Les rectangles $b$ et $d$ montrent deux dauphins gris et blancs, au contour noir (rehauts rouges et jaunes), affrontés de part et d'autre d'un cratère à godrons (noir, blanc et gris) d'où jaillit un petit jet d'eau. Sous chaque dauphin est placé un poisson rouge el rose (rouget?) regardant vers l'extérieur du panneau. Le fond laissé libre est décoré de motifs trapézoïdaux à traits chenillés (voir supra, p. 158). D'un rectangle à l'autre, quelques légères différences apparaissent dans l'arrangement de ces éléments el le rendu des animaux. Le style bien entendu reste le même, caractérisé par une schématisation extrême. On signalera en particulier le traitement du cratère du rectangle b, le mieux conservé (fig. 5 b). Le pied est figuré par une simple accolade; les anses ont disparu: : il leur plare, dans le prolongement des godrons latéraux, une sorte de pointe recourbée ${ }^{17}$.

Reclangles $e, f, g, h$ (fig. 1) : soulignés intérieurement par un filet, noir, ils sont couverts de deux Lypes de décors géométriques, à base de peltes ou d'osselets qui se correspondent, selon les diagonales.

Rectangles e et g : paires langentes de peltes adossées, alt ernativement couchées el dressées. Les peltes, dessinées par un filet blanc, enferment une pelte emboîtée à dominante grise, cernée de noir. Le plus souvent, une tesselle rose marque le point de contact des paires de peltes. Les intervalles cordiformes déterminés par la composition sont délimilés par un filet noir (fig. 7).

Rectanges $f$ et $h$ : osselets adjacents, au contour noir (f) ou hlanc (h) chargés d'une figure emboitée, généralement bordée d'un filet blanc (f) ou noir (h). de couleur variable (rouge, rose, jaune, blanc, noir). La plupart des osselets sont frappés en leur centre d'une harre noire ou blanche (fig.. 7).

Les petits carrés qui cantonnent le carré central (fig. 1 et 8) portent tous le même motif, constitué de deux éléments triangulaires ornés de nervures el ì base en festons, placés de part et d'autre d'un filet médian disposé selon la diagonale de la case. A premierre vue insolite, ce molif paraît correspondre, ainsi que nous l'avions déja suggréré18, à une représentalion de deux coquilles affrontées. Quant aux triangles situés sur le pourtour du champ. ils enferment un triangle emboilé, délimité de façon plus ou moins régulière, par un filet rouge a denlicules en quatre-tesselles, tournés vers l'extérieur.

Il est clair que cette mosaïque de Maubourguet relève d'un programme décoratif unitaire ; centré sur l’iconographie d'Océan, parfaitement mis en évidence par l'organisation axiale et symétrique de l'espace, ce programme était bien approprie, comme on l'a souligné plus liaut, a un pavement de bassin. Il est également manifeste, dapres la nature des différents éléments décoratifs at la façon dont ils ont été traités, que l'on a affaire à une cuvre provinciale qui doit ètre classée parmi l'abondante production des ateliers de

17 sur cette deformalion du cratere, voir (:. Barmatra, op. ril.

18 C.. Baturetse, op. ril. 
mosaïstes du Surl-()uest de la Ciaule durant l'Antiquite tardive Celle derniere affirmation mérite sans aucun doute des explications et esera le sujet de notre derniere partie.

La tete d'Océan retiendra d'abord notre attention. Celle divinite a elé somme toute peu représentée sur les pavements aquitains; outre Maubourguet, un seul exemple est actuellement connu : il s'agit de la mosaïque de Montréal-du-Gers, Le Glesia qui décorait une villa de la fin de l'Antiquité, partiellement dégagée au $\mathrm{x}^{\mathrm{e}} \mathrm{e}^{\mathrm{s}}$ ì̀cle ${ }^{19}$. Cependant, ces images ne sont pas aussi isolées qu'on pourrait le supposer à première vue. En effet, on retrouve à Jurançon Ponl dOly (Pyrénées-Atlantiques), soit à une soixantaine de kilomètres de Maubourguet, deux pavements figurant des divinités marines en buste, l'une un Neptune, l'autre difficile à identifier ${ }^{20}$. D'autre part, il convient de signaler l'existence, dans un contexte géographique assez proche, quoique en Narbonnaise, d'une autre mosaïque d'Océan, celle de Saint-Rustice, près de Toulouse ${ }^{21}$. Compte tenu du nombre limité de mosaïques d'Océan postérieures au II $^{\mathrm{e}} \mathrm{s}$, , dans les provinces gauloises, la forte concentration de ces images dans le Surl-Ouest de la (iaule semble un phénomène intéressant à souligner ${ }^{22}$.

19) La principale bibliographic est mentionnée dans G. IAFAYs, Inventaire des mosaiques de la Gaule, I, Narbonnaise el Aquilaine, Paris, 1909, no 457. Voir aussi les articles de l'abbe Cazacras dans l'Appel au Peuple (9 octobre, 29 et 30 octobre $1 \times 87$. Deux dessins alors publies, l'un de M. Rancé (Bull. de la Société de Borda, 12, 1887, p. 249-268, fig. 2), l'autre d'A. Iucamin Bull. arch. du Comili, 8, 1890, face p. 38, pl. I!. Postérieurement, ce pavement, quoique souvent mentionné par les spécialistes de mosäques inotamment L. Foccater, La maison de la procession

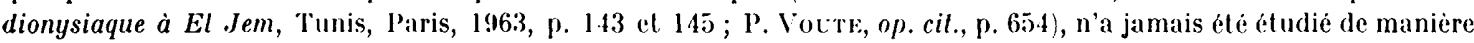
approfondie.

Parmi le matériel recueilli sur le sile, sont mentionnées principalement deux monnaies provenant des déblais d'une salle de la villa : la premiere, un grand bronze de Trajan ou d'Hadrien, la seconde, un petit bronze d'Hélène, mère de Constantin, de l'atelier de Treves, appartenant à unt importante série frappée entre 324 et 330 (Lale Roman Bronze Coinage, A.D. 324-498, London, 1960, I, p. 4, Treveri $n^{\circ} 47$. La datation proposée à la suite de ces trouvailles est imprécise et peu claire. I.es caractéristiques générales du décor de l'ensemble des cinq mosaïques mises au jour dans la villa, inciteraient à écarter une datation antéricure au ive s. : cf. ultérieurement, Recueil, $I V, 2$, en préparation.

20 Recueil, IV, 1, no 153 (pl. XC, X(II, XCIII) el no 15.1 (pl. XCirV el X(iVI). Datation proposée : Ive s. au plus tòt (cf. observations, ibid., p. 164).

21 Découvert en 1833, le pavement est mentionne par (i. Lalaye op cil, n०376, planche). Par la suite, le principal article consacré à cotte mosaïque est celui de R. I.izop La mosaïque de saint-Rustice el ses inscriptions,

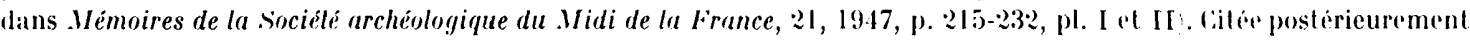

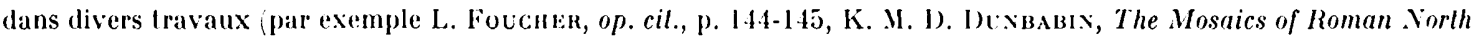
Africa, Oxford, 1978, p. 150, note 63, cette mosaïque n'a jamais fait l'objet d'une étude détaillée.

L'on ne dispose d'aucun renseignement précis sur le matériel trouvé au cours des fouilles. Dans les articles anciens, le pavement a élé altribué à des époques diverses : II ${ }^{\mathbf{e}}$, II ${ }^{\mathbf{e}}$, Ive $\mathrm{s}$. Il nous semble impossible, en raison du style de la mosaïque et de certains délails iconographiques, de retenir une datation antérieure au Ive s. On évoquera en particulier l'arrangement de la chevelure de Thétis 1R. Lizop, op. cit., pl. III ; sur les représentations mylhologiques (qui accompagnent la face d'océan, voir infra, : les cheveux de la déesse sont fixés par un cabochon central bleu et par des rangs de perles blanches, l'un encadrant le visage, l'autre partant également du cabochon, mais en arrière du front. Cette disposition de pierres précieuses dans la chevelure suggiere plusieurs rapprochements avec des representations de Vénus voire de néreide, Victoire, sur les pavements africains de la fin du we s. et du ve s. : ainsi a Sétif (J. Lasscs, Vénus Marine, dans Colloque mosaïque gréco-romaine I, Paris, 1965, p. 175-169, fig. 3 et d, vers 400),

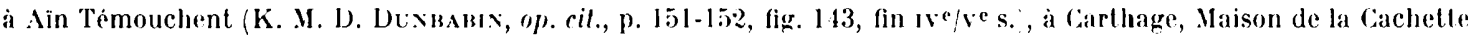
ibid., p. 158, fig. 150, fin we, debut ves., ; on mentionnera egalement un aulre pavement de larthage (Sidi Bou Saïd), conserve au British Museum (K. P. Haxs, Calalogne of the Greek, Elrnscan and Roman Paintings and Mosaics in the British .Museum, I.ondres, 1933, n" 59 , p. 149 , fig. $16 \times$, iv-ve s. ?. Celle disposition de pierres pricieuses pourrait avoir éte influencée par le lype de diademe impérial mis a la mode par Constantin vers 324 (cf. Age of Spirituality, Lale Antique and Early Christian Art, Third to Seventh Cienlury, New York, 1979, no 10, p. 16-18:.

22 Les autres representations d'océan connues dans les provinces gauloises paraissent se situer entre 150 et 250 . On notera en particulier la presence de ce theme sur Irois pavements de Vienne, dont deux noirs et blancs et un polychrome (cf. J. Laxcha, Recueil général des mosaïques de la Gaule, III, Narbonnaise, 2, Vienne, Paris, 1981, 


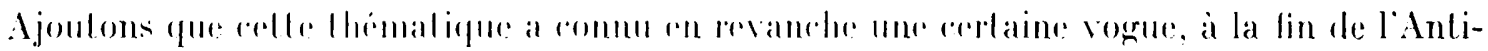
quité, dans d'autres provinces de l'rmpire romain d'ocecident, par exemple dans la Péninsule Thérigue ${ }^{23}$ at en Angleterre"24.

A. Maubourguet, le mosaiste a placé la fare d'orean, seule à l'intérieur d'un panneau carré, au centre du lapis. Lne disposition analogue se retrouve sur d'autres mosaïques d'ocean ${ }^{25}$. mais il est eviclent que re principe general une fois adopté plusieurs possibilités étaient offertes, selon lorganisation d'ensemble de l'espare. Alors qu à Montréal-du-fiers, la solution choisie est particulierement simple dans la mesure où la títe sótale dans un (hamp carré (rnviron 1.80$)$ m de (coté) uniquement siparé des murs par une bordure et par une bancle en marbre blane, a Vaubourguel, au contraire. on se trouve en présence diune organisation extremement complexe; aucun exemple précis de comparaison n’a élé repéré pour le système de composition centrée utilisé. Le rapprochement le plus suggestif est fourni par une mosaïque de la villa de Brading. dans l'ìle de Wight ; on retrouve le mème type re composition centré avec croix en sautoir inscrite. mais celle-ci n'est pas enlarée à un rarré sur la pointe. comme à Vaubourguetét.

Comparé aux autres representations de tites d'océan actuellement connues, l'image de. Maubourguet suggère plusieurs remarques. Premierement, il apparail que cette représen-

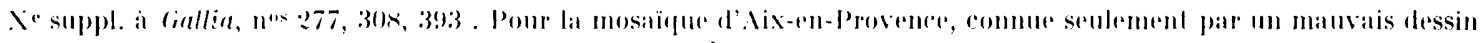

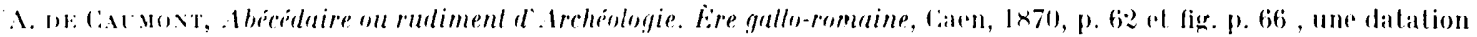

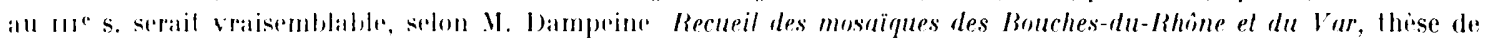

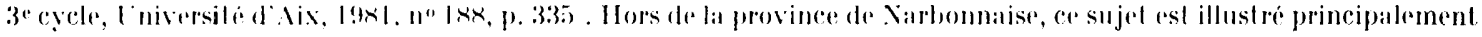

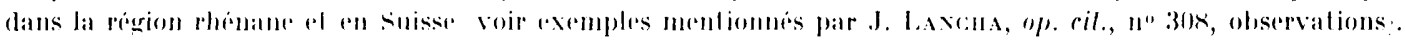

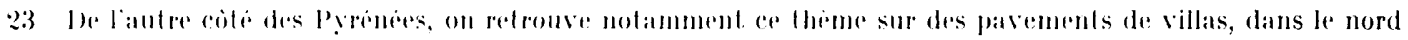

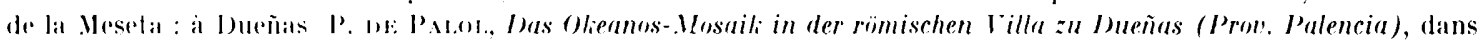
Malrider Milleilungen, Heidellerer.

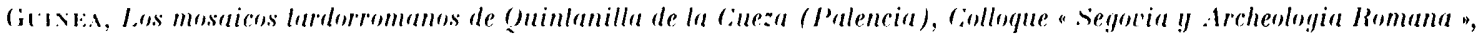

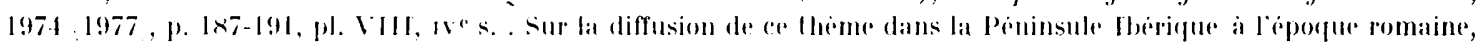

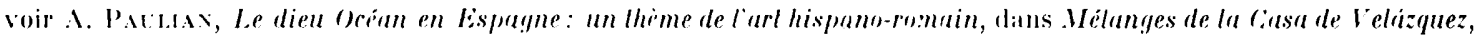
Xi, 1979, 1. $115-133$.

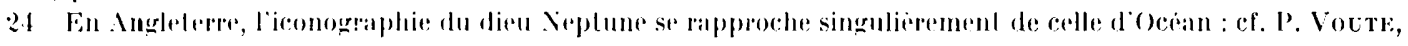

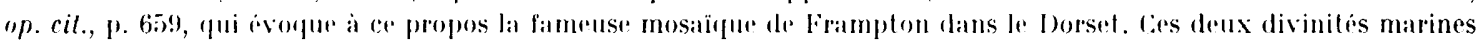

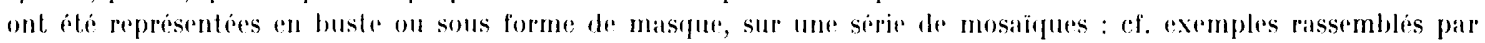

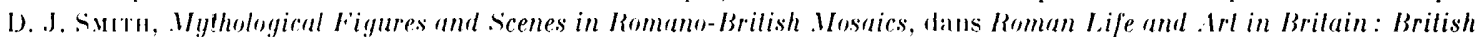
Archaeolegical Reports 11 i , 1977, p. 1:21-125. Parmi les l reize pavements ciles, dix sont dates du 10 s. el appartiennent a des villas concentrees daus le Sud de l'Angleterre.

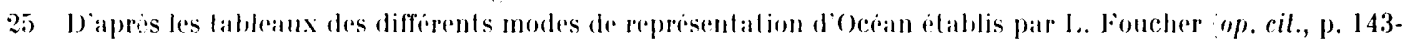
1.15 , cetle disposilion correspond an lype B, 2". Selon le chassement propose par P. de Palol op. cit., p. $215-217$, notre tapis serait a rallacher au second groupe. les patrements oi ocian est figure seul, au centere du champ, à l'interieur

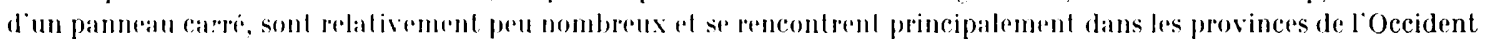

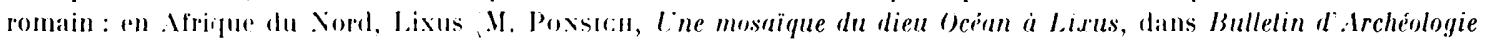

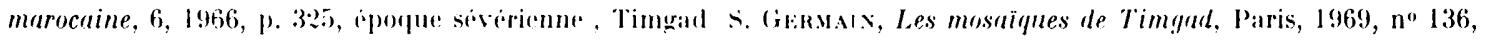

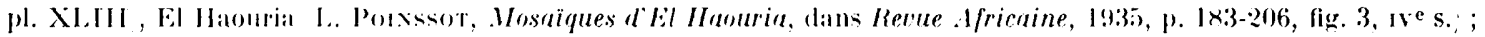

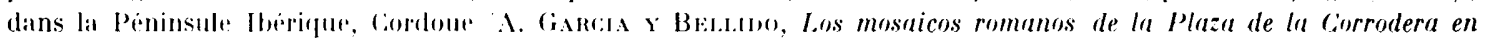

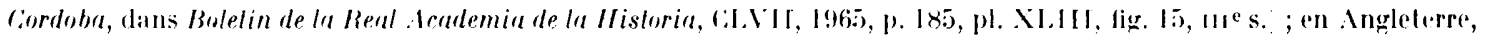
Varulamium 1). J. simtu, Roman . Wosaics in Brilain before the Fourlh Century, dans Colloque mosä̈que gréco-romaine Il,

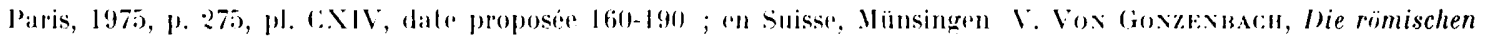

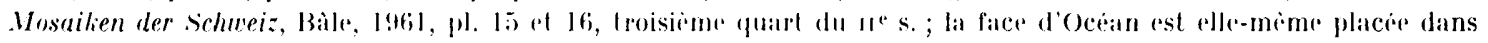
un médaillon circulaire.

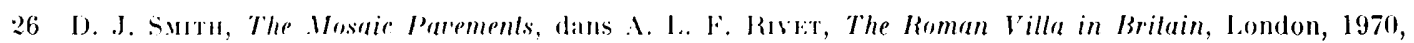
p. $91-94$, pl. $3-8,1 N^{\circ}$ 

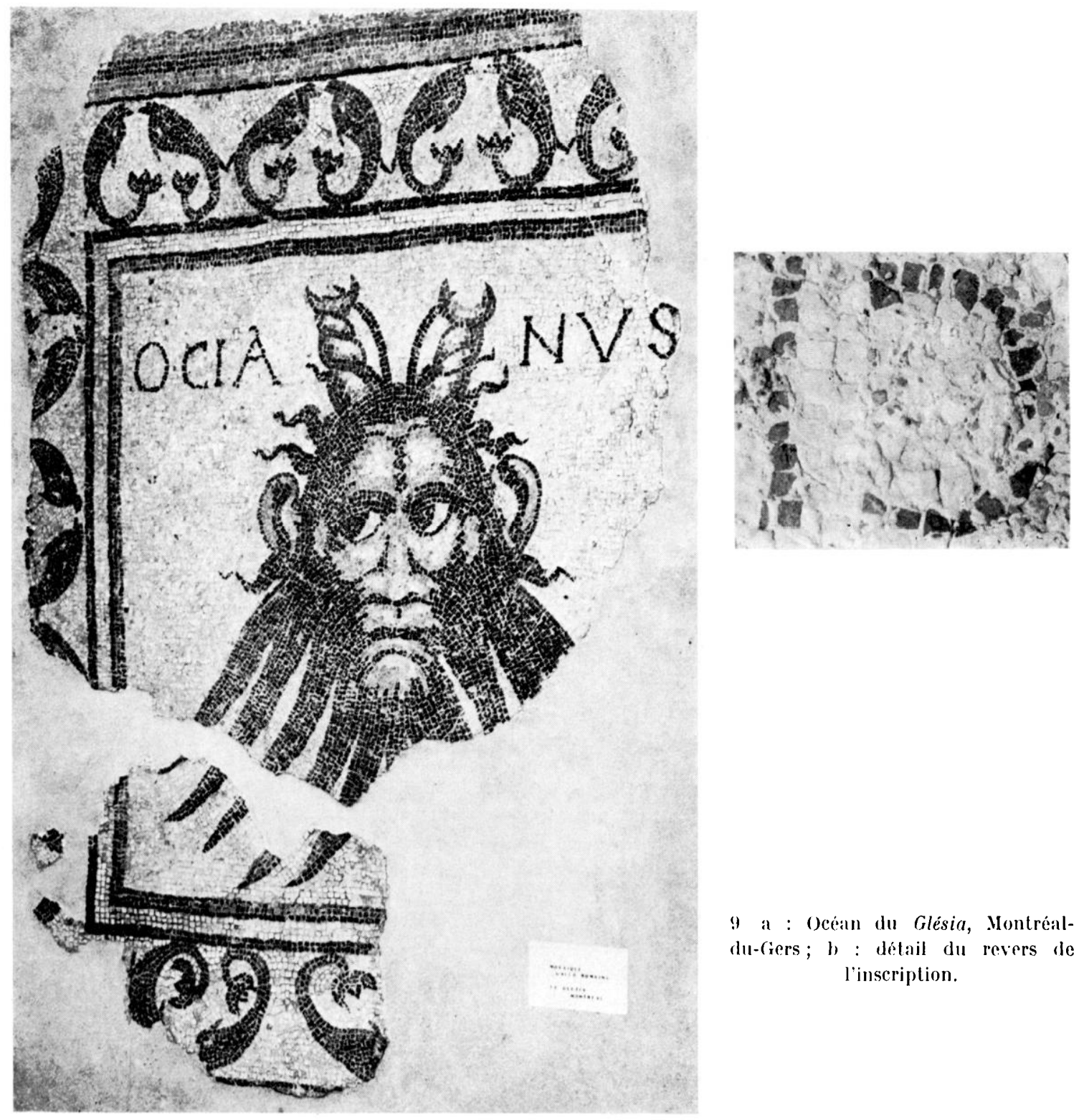

9 a : Océan du Glésia, Montréaldu-Gers; b : détail du revers de l'inscription.

tation, tout en réunissant les caractéristiques iconographiques d'Océan ${ }^{27}$, n'est directement comparable a aucune auter image de ce dieu, tant dans un contexte aquitain que dans le cartre plus vaste des provinces de l'empire.

Malgré la présence l'attribuls communs, pinces, antennes, vaste chevelure, la tête d'océan de .Iontréal-du-liers (fig̣. 9) $)^{28}$, noffe aurun parallèle précis aver notre mosaïque :

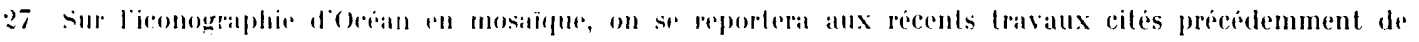
L. Fonchere cf. note 19, de P'. de P'ilol ef. note 23 , et de P. Voute cf. note 14 . Quelques nouvelles remarques de 1. Foucher a ce sujel, dans son arlicke, Sur licomographie du dieu Ocium, dans Ciaesarodunum, 10, 1975, p. 48-52.

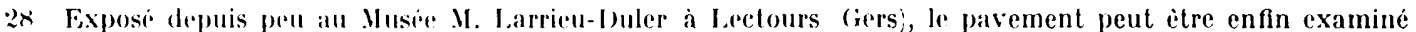
dans son aspect originel, a la suite de la restaumation effectue en 19x0 par J.-L. Laffont (Atelier de Restauration 
d'une part, le rendu des divers éléments n'est pas comparable, d'aulre parl, a Maubourguet, s'ajoutent d'autres motifs décoratifs, dauphins, coquille, bouton de fleur, lesquels contribuent à donner une allure très particulière à la face du dieu. On nous permettra d'ouvrir ici une parenthèse pour souligner l'intérêt de cette mosaïque de Montréal-du-(iers : l’image du dieu est en effet accompagnée, ce qui est exceptionnel, d'une inscription en caractères latins : OCIANCS (fig. 9 a ${ }^{29}$. Cette lecture jugée erronée par certains au moment de la découverte ${ }^{30}$ semble devoir itre retenue après examen du revers de la mosaïque (fig. (9 b)) ; on précisera que cette notation phonétique correspond à une confusion entre les voyelles $i$ et $e$, phénomène bien attesté dans le latin vulgaire de l'Antiquité tardive. en Ciaule, tant, par les inscriptions que par les sources textuelles ${ }^{31}$.

Si l'on considère l'autre mosaïque d'Océan connue dans le Surl-()uest de la Ciaule. ceelle de Saint-Rustice (fig. 10), les différences avec l'Océan de Maubourguet sont encore beaucoup plus manifestes, à la fois à cause du style el de l'iconographie générale du pavement. Malgré les nombreuses restaurations modernes et l'etat lacunaire de certaines parties, on ronstate que la face du dieu, polychrome, également carartérisée par des pinces de crustacé, des antennes, une chevelure abondante et des dauphins jaillissant de la harbe ${ }^{32}$, releve d'une esthétique opposée, dans la tradition des grandes tîtes majestuenses d'Afrique du Nord ${ }^{33}$. Les sujets figurés qui sont associés à Oréan situent en outre ce lapis dans une tout

de la ville de Toulouse; qui a fait disparaitre les restitutions peintes de la fin du xixe s. Plusieurs délails, pon parliculier les pinces rouges dessinés par des torsades ombreses surmonteres d'un motif an forme de croissant, semblent siture

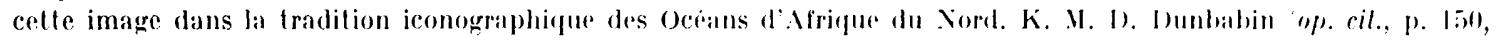
note 63 ) mentionne simplement a propos de cel ocian de Monlebal quiune inllunce africaine est possible.

29 A part. Wontreal, Le lilésia, un seul pavement, semble-t-il, presente une inscription en latin, identifiant

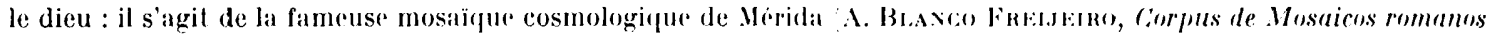
de España, I, Mosaicos romanos de Merida, Madrid, 1978, 117 , pl. 39.

30 Bullelin de la Sinciple de Borda, 12, 1887, p. 260.

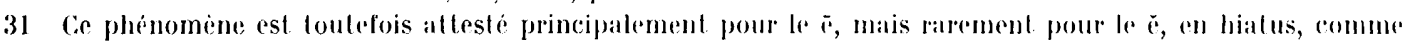

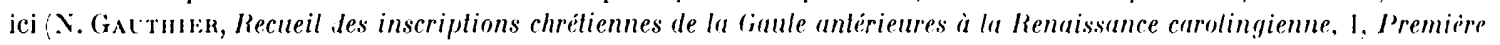
Belgique, Paris, 1975, p. 62-63:. Dans le latin vulgate, la notation I ou E . quand les voyelles sont en hialus .

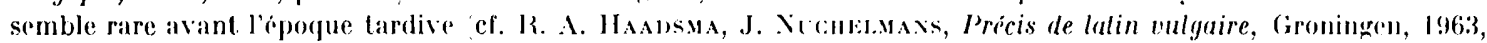
p. 23). La graphie (ocianus pour Oceanus paraît par ailleurs exceptionnelle; un seul exemple a elé repéré sur une inscription de liome : C.I.L., VI, 3, no 21919 . 11 convient de preciser que celte inscriplion n'est connur que par des manuscrits, ce qui laisse subsister un doute sur l'authenticile de la graphie. Oulre les temoignages ipigraphipues, on evoquera le lexte bien connu de consentius, grammairien de Narbonne, qui releve che\% les Gaulois au v? siecle, une confusion entre les sons i et e Consentii, Ars de barbarismi el melaplasmis, ed. M. Nirdermann, Xinchatel, 1937, p. 15, \$15).

32 L'Ocean, expose successivement au Musere des Augustins al au Musée Saint-Iiaymond a Toulouse, se trouve a l'Atelier de Restauration de la ville de Toulouse, ou il sera prochainement restauré par J.-I. I.affont. ()n ne distingure. a l'heure actuelle qu'une seule pince, sur le cote ganche du front; les antennes et les dauphins sont lacunaires. On remarquera aussi les oreilles, materialisees par une sorte de cornet d'oi surgit un protome d'animal marin pour des exemples comparables, cf. V. Vox Goxzexbacil, op. cil., pl. 16 et p. 138-139;.

33 A propos de cet Ocean de saint-Rustice, K. .. I). Imubabin (op. cil., p. 150, nole 63; signale simplement

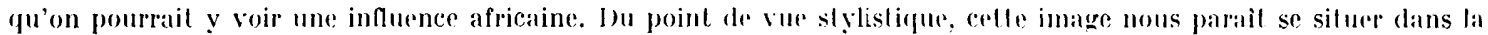
lignée des grandes tètes expressives et dramatiques, comme celles de Thimetra l.. Foucul:n, Thermes romains des

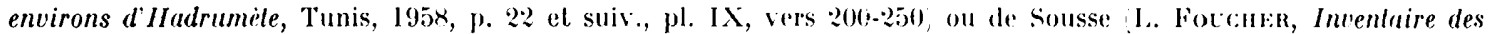
mosaiques. Sousse, Tunis, 1960, no 57041 , milieu du ${ }^{\mathrm{e}} \mathrm{s}$. En cerlain nombre doblements confirme, semble-t-il, cette

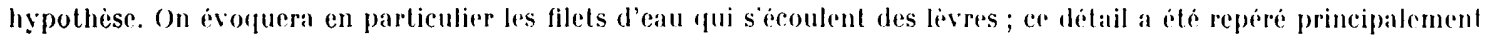
sur des représentations docean en Ifrique du Nord : a Themelm, a sousse ; cf. supra, a Althihuros M. Fxxalfir,

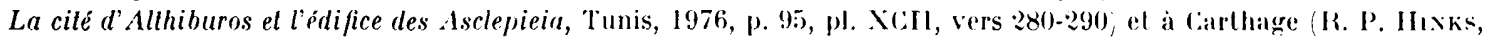
op. cit., n० 15, p. 74-75, pl. XXVIII, Ire s. ?. Le lissu tendu par les amours suggere igalement des paralleles arece les mosaïques d'Afrique du Nord voir infra, note 34 : 


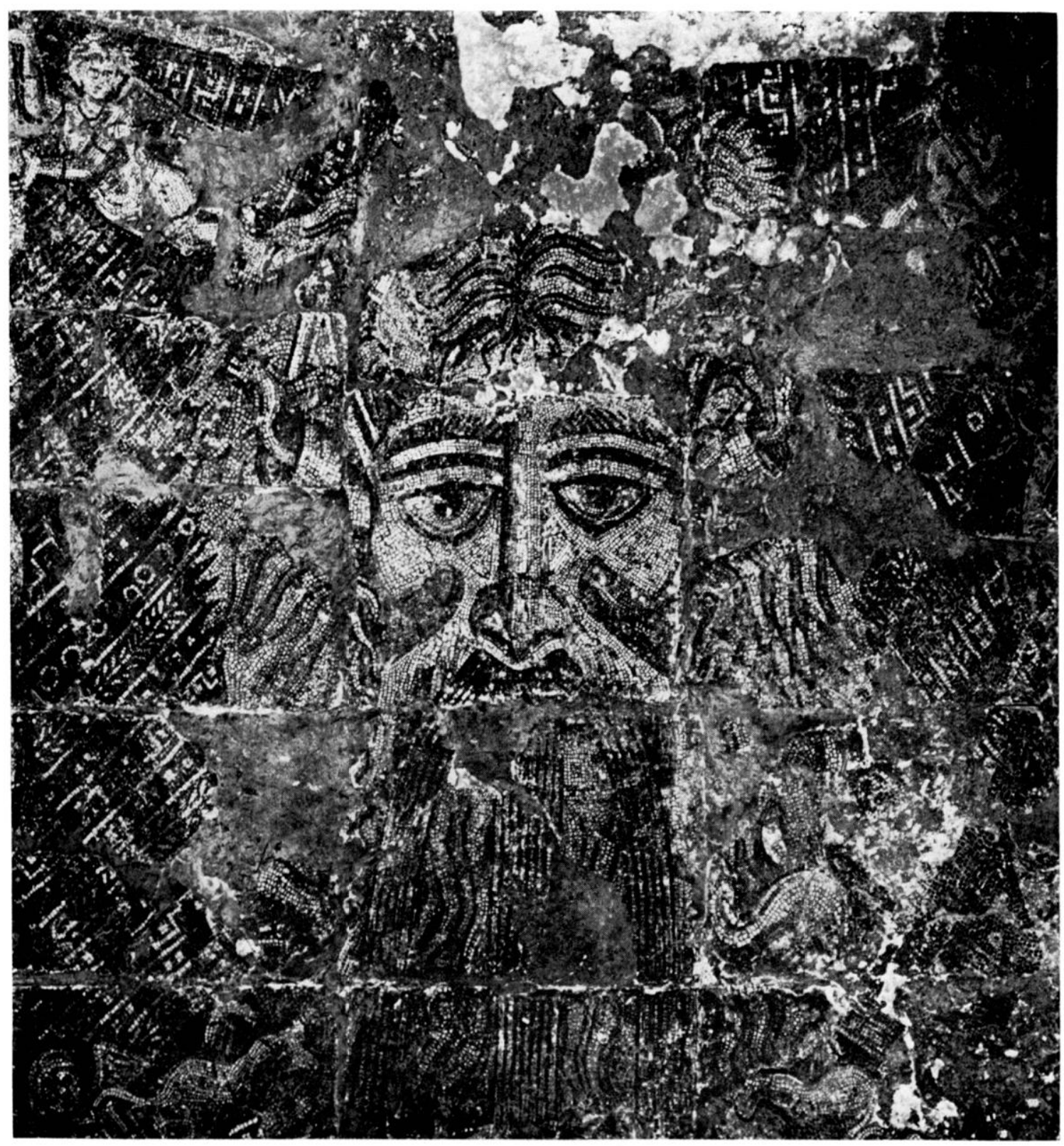

10 Océan de Saint-Rustice.

autre ambiance culturelle : (quatre amours ailés portant un bracelet à chaque poignet et à chaque bras et un collier orné d'un pendentif autour du cou (bulla), maintiennent les angles d'une étoffe richement décorée qui sert de toile de fond à la teite du dienos; d'autre part,

34 Colle composilion mapelle singulieremenl certaines represendations de linus marine sur le's pavements

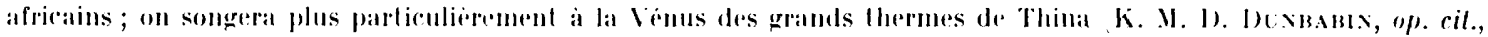
p. 105-106, fig. Ix, fin du ule s... C.elle facen de mettre con valeur l'image de la divinite. peu habituelle dans les mosaïques

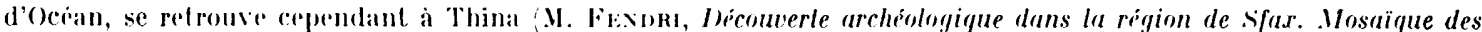

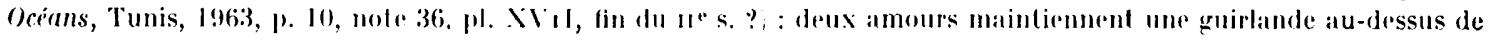
lat têtu diru. 


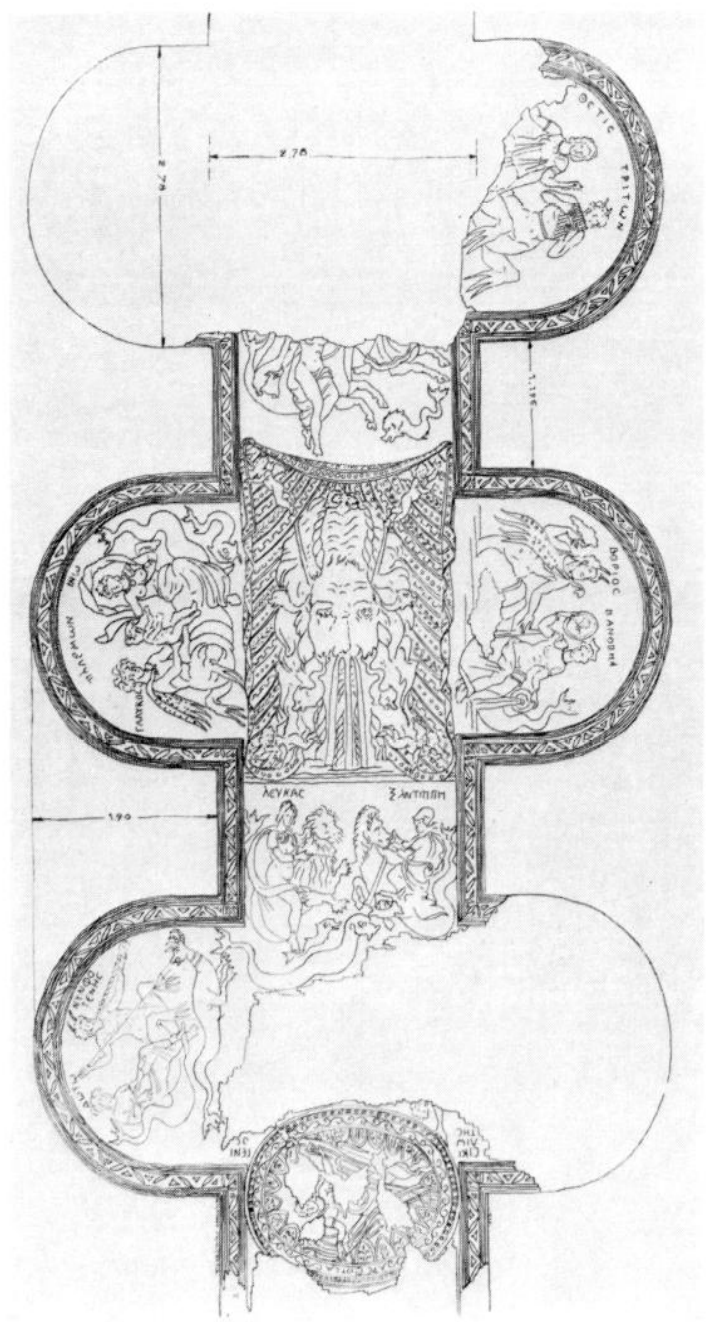

11 Sint-Ruslice:

dessin de l'ensemble du parement

(d'apreis R. I.izop.

tout le reste du pavement est oceupé par divers groupes de personnages mythologiques appartenant au panthéon océanique ou marin, parfaitement idlentifiables grâce aux inscriptions en lettres grecques qui les accompagnent (fig. 11) ) $^{35}$.

Lorsqu'on abandonne le: Sud-Ouest de la (iaule pour examiner l'importante série de mosaïques figurant la face d'Océan, les éléments de comparaison restent très limités, non significatifs. Il est évident que le pavement de Maubourguet, par ses traits stylistiques, se rattache aux représentations tardives d'Océan rencontrées lans la partie occidentale de l'Empire, notamment dans la Péninsule Ibérique, en Afrique du Nord et en Angleterre ${ }^{36}$,

35) La mosaïque recoure une gratude salle rectangulaire $13 \mathrm{~m} \times 2,78 \mathrm{~m}$; a alsides. Voir K. Lizop, op. cit., p. 223 et suiv, et pl. I. Sur l'attachement de l'aristocratie provinciale a la langue grecque, dans l'occident romain,

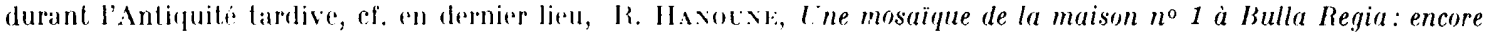

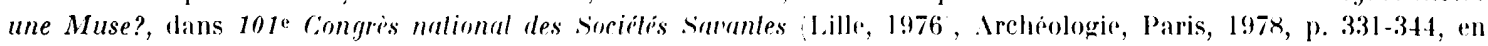
particulier p. 343.

36 En ce qui concerne la Pininsule Ibirique et l'Anglelerre, voir supra, notes 23 el 2.4. Fn Afrique du Nord, ce thème, surtout en faveur aux n-me s., est encore illustri an we s., voire mème un peu plus tard, sur divers pavements : Carthage maison d'Ariane, El Haouria, Ain Temouchent et cherchel voir tableaux avec réferences de 
mais il n'en demeure pas moins que cette image reste isoléc, sans répondant direct. On est donc certainement en présence d'une production régionale, sinon locale, exécutée par un mosaïste qui a donné libre cours à son imagination et à sa fantaisie. Il n'empêche que l'artisan - ou le commanditaire connaissait liconographic canonique du dieu Océall. Tous les détails iconographiques communément attestés ont été repris, mis en valeur : pinces, antennes, barbe limoneuse, dauphins ; à propos de ces derniers, on remarquera leur taille démesuréc et leur corps particulièrement étiré qui sort curieusement du nez d'Océan, et non pas de la barbe ou des cheveux. comme il est habitue ${ }^{37}$. L'aspect étrange, mi-humain mi-animal, commun à la plupart des images de ce dicu, a été accentué par la disproportion du visage et l'extreme simplification des traits. Toutefois, la principale innoration du mosaïste a consisté à placer au-dessus de la tête du dieu, une coquille agrémentéc d'une feuille. L'expression de gravité sévère ou dramalique, propre à de multiples têtes d'océan ${ }^{38}$, fait place ici à une expression de gravité cocasse.

La face d'Océan, sur la mosaïque de Maubourguet, est entourée d'éléments aquatiques, dont quelques-uns, dauphins et coquilles, étaient déjà associés à la représentation du dieu. Le répertoire utilisé, à lexception des motifs trapézoülaux couverts de traits chenillés, est bien attesté sur les parements tardifs du Sud-Ouest de la Gaule. I.es dauphins en particulier apparaissent fréquemment, employés de diverses manières, mais toujours rendus schématiquement, caractérisés en général par une queue trifide rouge el des rostres prononcés. On les rencontre par exemple, associés à des poissons, des crustacés et des poulpes, sur une série de mosaïques marines, décorant pour la plupart des bassins ; on évoquera plus spécialement, en raison de la disposition héraldique des cétacés, le pavement du bassin triconque

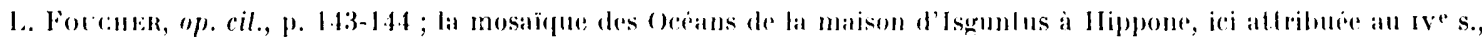
doit efre placere entre 210 of 260, dapres les recents sondages de .J.-P. Morel : sur ce point, cf. notamment

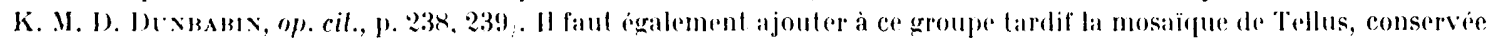
a l'Antiquarium de Carthage fef. P. Vocte, op. cil., p. 666, fig. 16. Signalons enfiu que focéau de la maison des

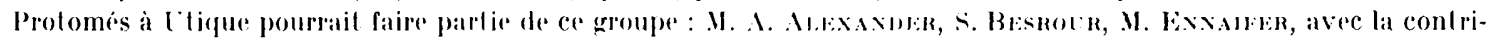

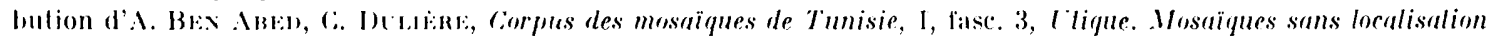

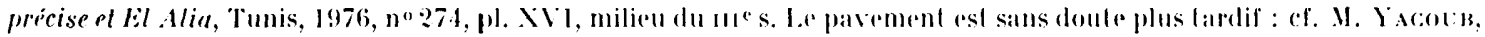

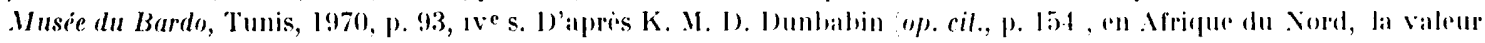
apot ropaïgue de l’image du dien ne devient nettrment explicile qu'a partir du we s.

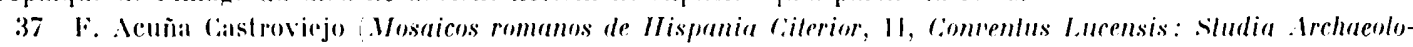

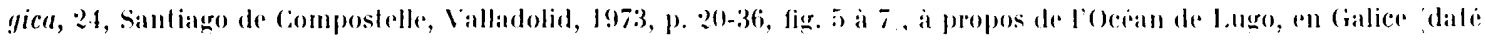

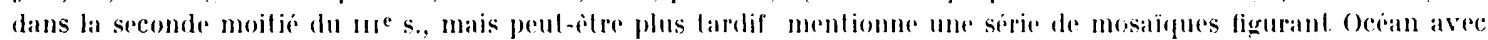
deux dauphins sechappant de la barle voire des oreilles : parmi les exemples ciles pour le me s., certains pavements

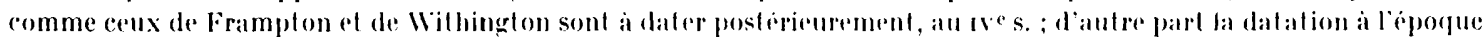

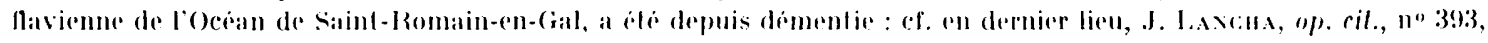

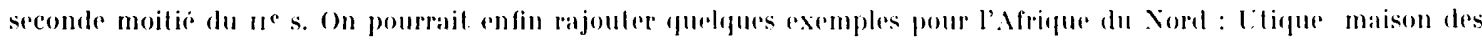

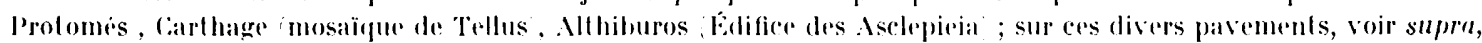

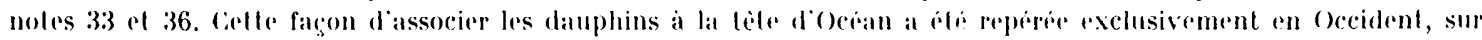

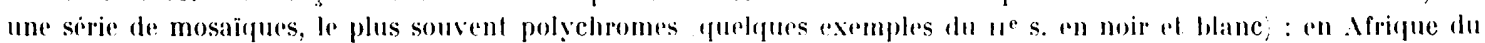

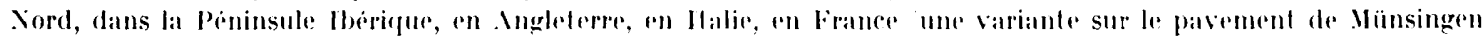

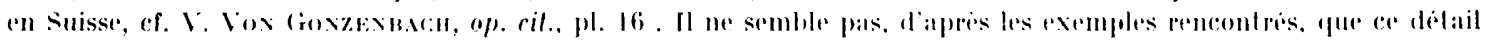

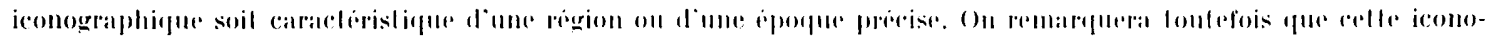

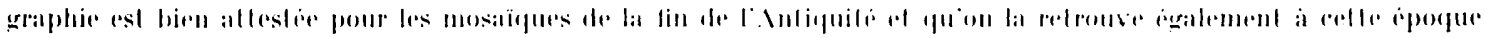

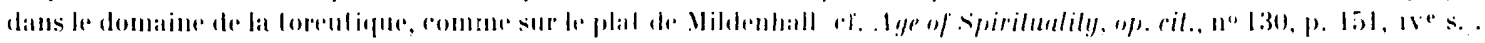

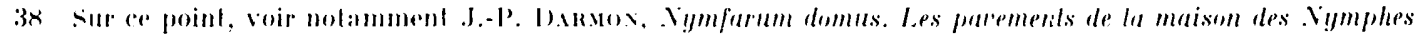

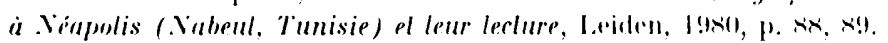


de Jurancon, Pont d'Oly ${ }^{39}$. Ce mode de représentation du dauphin par paire d'éléments affrontés, se retrouve sur plusieurs tapis, notamment à Nérac et à Bordeaux ${ }^{40}$ : dans les deux cas, les dauphins sont séparés, comme a Maubourguet, par un vase. On notera aussi qu'une frise de paires de dauphins constitue la bordure de la mosaïque d'Océan de IIontréaldu-Gers (fig. 9 a). Cette forme d'utilisation du motif semble, par contre, peu courante en Aquitaine ${ }^{41}$.

Les poissons sont également figurés sur de nombreuses mosä̈ques de cette région. kin grande majorité non identifiables, ils appartiennent à la même catégorie de répertoire que les dauphins, auxquels ils sont souvent associés, dans les représentations de mer poissonneuse $e^{42}$. Il convient cependant de spécifier que les sujets aquatiques des rectangles a et c (fig. ja et c) qui regroupent des poissons et des canards, type de scène banale dans la mosaïque romaine ${ }^{43}$, ne trouvent aucun parallèle sur le plan local. On remarquera simplement. que des canards apparaissent sur quelques tapis, comme remplissage d'un élément géométrique (octogone, carré) ${ }^{44}$. Parmi les autres motifs attestés a Maubourguet, il faut enfin prêter attention à la coquille: : il s'agit en effet d'un ornement que les mosaïstes du Surl-ouest de la Gaule ont fréquemment utilisé, sous des asperts divers, l'assoriant parfois comme iri à des sujets marins ${ }^{15}$.

A l'inverse de la romposition rentrée qui semble ôtre un hapar (voir supra. p. 162). les motifs géométriques qui décorent les rect angles (osselets arljacents, paires de peltes adossées: alternativement couchées et dressées), correspondent à des trames souvent rencontrées en Aquitaine, durant I'Antiquite tardivet6. On notra la facon particuliere dont sont dessines

39) Recueil, IV, 1, 11" 15, pl. Xil.

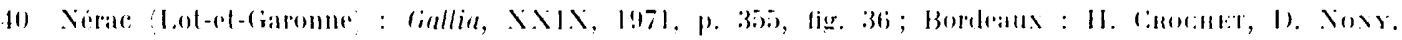

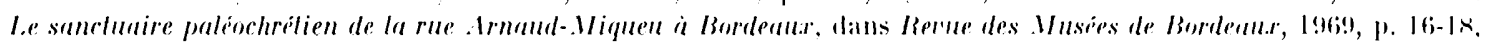

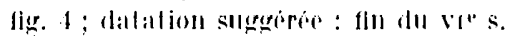

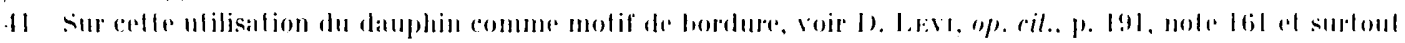

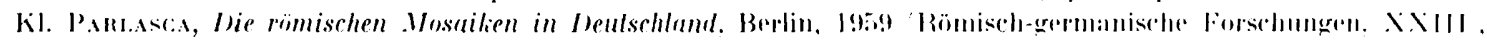
p. 10, nole 4 (1) fll. 10, I.

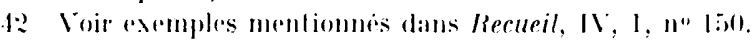

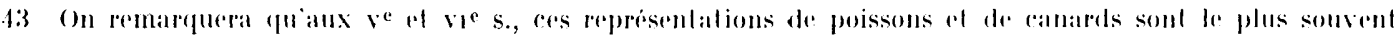

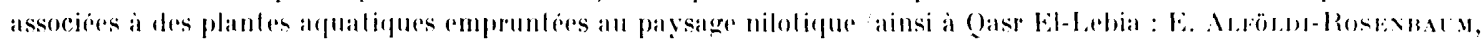

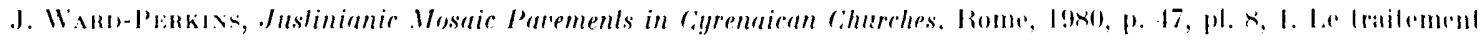

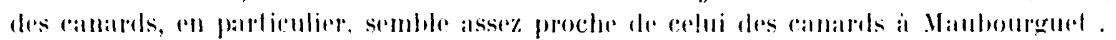

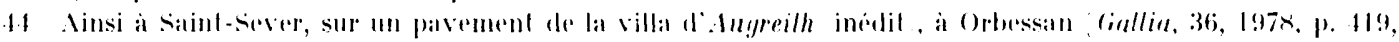

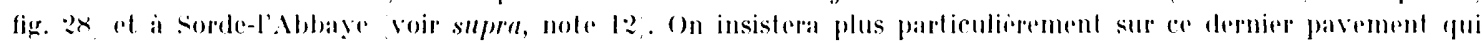

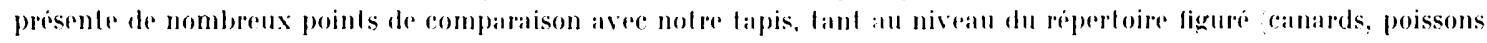

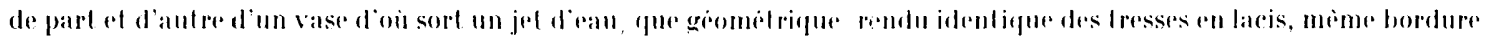
en filets denticules rouges.

li) Voir par exemple Recueil, I, 1, n" lis, observalions.

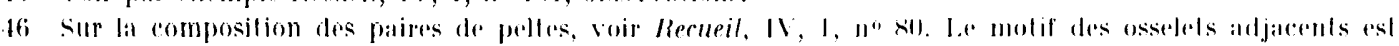

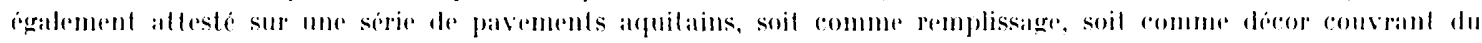

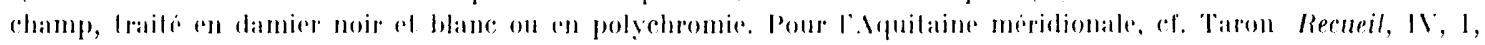

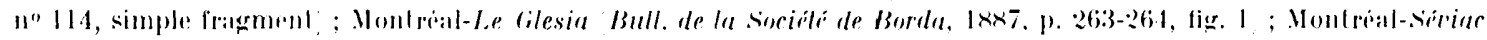

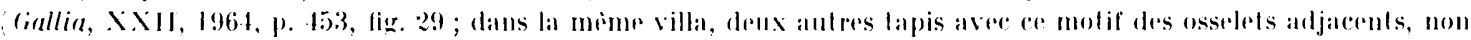

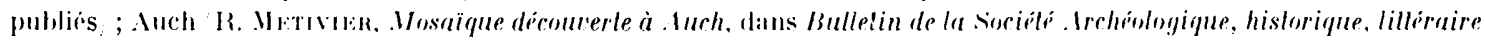

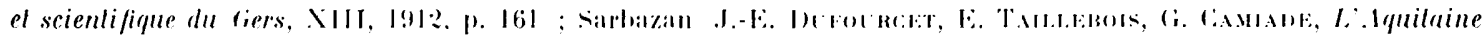

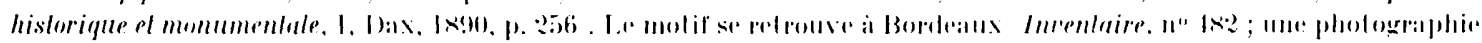

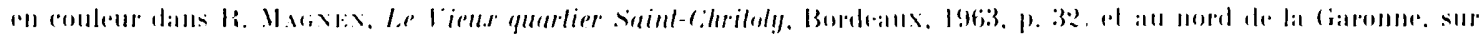

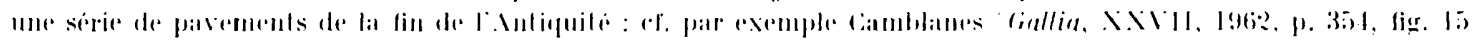
et Jouplate fiallide. IX, 1951, 1. :210, figr. I1. 
la plupart des motifs (fig. 1 et 7 ) : contour blanc, filet intérieur noir délimitant une figure emboitée colorée. Attestée sur un nombre limité de pavements du Surl-(Ouest, cette manière de tracer certains motifs géométriques, tels que les peltes et les osselets, se retrouve princi-

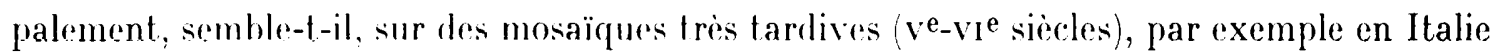
du Nord et en Afrique du Nord ${ }^{17}$.

Cette mosaïque illustre donc parfaitement la production des ateliers de mosaïstes du Sucl-()uest à la fin de l'Antiquité. Le répertoire, on vient de le voir, se rattache à un fonds iconographique commun à une série de pavements. Sont également caractéristiques le choix des matériaux ${ }^{48}$ et le style très libre marqué par la recherche de linéarité et la tendance à réduire le décor figuratif à des volumes géométriques. Autre trait spécifique de cette production, sans nul doute le plus intéressant, c'est la part d'improvisation, de créativité du mosaïste par rapport aux formules recues. L'image d'Océan constitue à ce titre un exemple remarquable. Toutefois, comme on l'a déjà évoquét ${ }^{49}$, l'originalité dont témoignent certains pavements du sud-Ouest de la ciaule durant l’Antiquité tardive, ne saurait être envisagée comme un phénomène isolé : une esthétique nourelle, un besoin de créativité caractérise a cette époque la production artistique dans diverses provinces de la Méditerranée, notamment en Afrique ou P.-A. Férier observe "une rupture dans l'esthétique qui répond à la transformation de l'ansemble du monde méditerranéen. Rupture qui donne une vie nouvelle a des thèmes hérités de la mythologie traditionnelle ou empruntés à la vie quotidienne $n^{50}$.

Si le contexte général de la fouille et l'analyse stylistique permettent de situer avec; certitude ce pavement dans l'ambiance de l'Antiquité tardive, il semble en revanche très difficile, voire impossible, de fixer une date absolue. On rappellera que la fouille n'a fourni aucun élément précis de chronologie et que les documents de comparaison repérés dans un milieu géographique proche apportent des informations limitées, étant donné le caraclere ponctuel de ces rapprochements ot l'incertitude des datations, soit qu'il s'agisse de découvertes du xixe siècle comme à Nontréal-rlu-(iers, Le Glésia, et à Saint-Rustice, soit que la fouille n’ait permis de fixer qu'un lerminus ou une chronologie relative.

A première vue, l'extrême schématisation des sujets figurés, les malarlresses d'exéculion seraient en faveur d'une datation très basse, mais il ne faut pas oublier que des raractéristiques analoguess apparaissent sur de nombreuses mosaïques provinciales dès le

47 En Aquitaine, on a repere des osselets traites de maniere analogue uniquement sur une mosaígue de Bordeaux cf. supra, note 16 . Fn revanche, ce traitement est bien allesti a Ravenne, au vie s., sur plusieurs pavements : 1. Fartol.t, Pavimenti musini di Ravenna palencristiana, Ravenne, 1975, fig. 3, p. 17 ; fig. 8, p. 23 ; fig. 106, p. 208. be's pelles polychromes, alu contour blanc, se retrouvent également a Bordeaux, sur te meme pavement. En dehors du sud-()uest de la (raule, on observe les mimes caracteristiques dans le rendu des peltes, sur la mosaingue de la nef

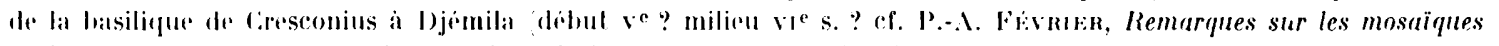

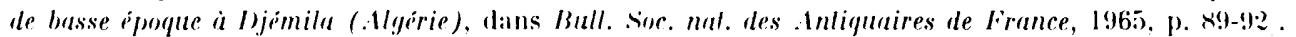

tr On notera en particulier lemploi de marbere gris bleute pour le rendu des corquilles al du bouton de fleur trifide; le mime materiau utilisi pour les mimes motifs se retrouve sur de nombreux pacements aquitains voir

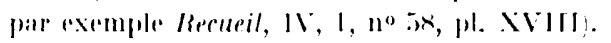

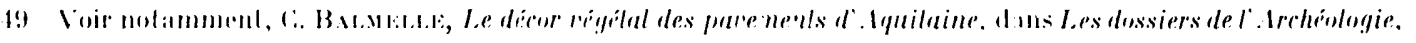

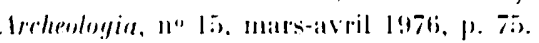

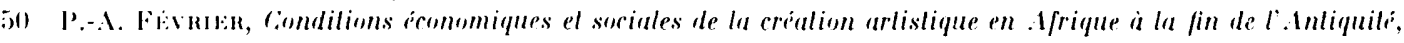

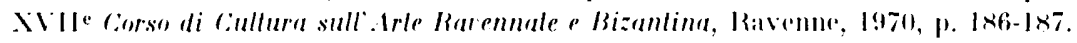


Ive siècle, par exemple en Angleterre ${ }^{51}$. Lin revanche, certains détails dans le lracé des éléments géométriques (lacis de tresses, osselets, peltes) semblent correspondre à des particularités techniques bien attestées aux ve et ve siècles, rlans l'ensemble du monde méditrerranéen. Dans ces conditions, on serait tenté de proposer pour le pavement de Maubourguet, une datation postérieure au $v^{\mathrm{e}}$ siècle. L'organisation de l'espace dans les panneaux rectangulaires décorés de canards et de poissons, inciterait également à relenir cette proposition ${ }^{\text {j2. }}$

Il resterait enfin à évoquer le poids de la tradition antique, dont témoignent tant les textes que les vestiges archéologiques, non seulement au $\mathrm{N}^{\mathrm{e}}$ mais encore aux $v^{\mathrm{e}}$ et $\mathrm{vi}^{\mathrm{e}}$ siè La survivance des thèmes mythologiques dans l'art décoratif. reflet d'une tradition classique restée vivante dans l'aristocratie riche et cultivée, et aussi dans les milieux plus modestes, constitue l'une des réalités de cette période complexe qu'est lintiquité tardive. comme a si bien mis en évidence, récemment, l’exposilion organisée par K. Weitzmann, au Metropolitan Yuseum de New York ${ }^{53}$.

Ainsi, cette mosaïque de Maubourguet, ou l'on releve a la fois une rupture dans l'esthétique et une continuité dans l'iconographie, sinscrit-elle parfaitement dans la production artistique de l'Antiquité tardive. On ajoutera que cette continuite thématique: ne semble pas purement formelle; l'image du dieu, cntourée d'animaux aquatiques el marins, pourrait avoir conserve sa signification originelle de pere de toutes les raux.

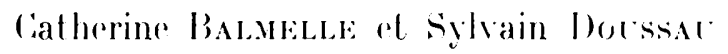

(C....R.S.)

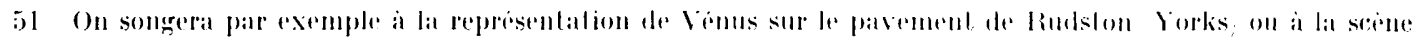

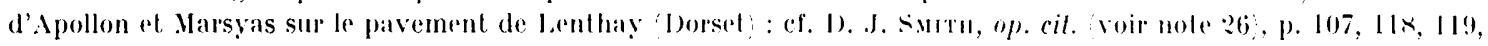
pl. 3.20 el 3.31 .

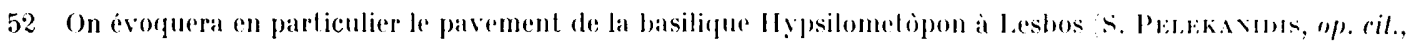

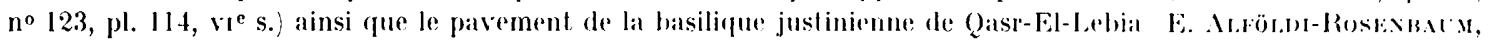
J. WARD-PERKIXs, op. cil., pl. 8,1 et 2 '.

53 Sur cette exposition novembre 1977-f6ivier 1978, voir Calalogue, Age of spiritulity cf. supra, nole :21.

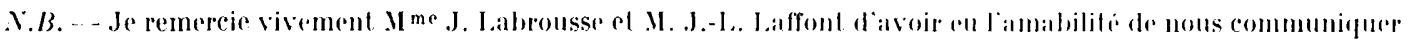

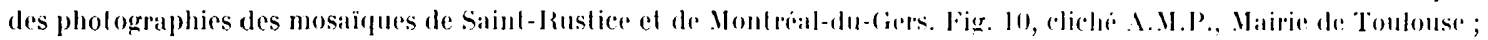

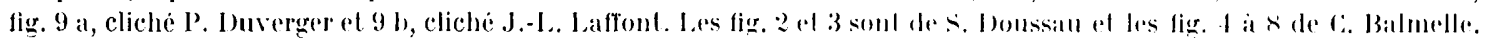

